\title{
Gestão da cadeia de suprimentos: análise dos artigos publicados em alguns periódicos acadêmicos entre os anos de 2004 e 2006
}

\author{
Supply chain management: analysis of papers published \\ on some journals between 2004 and 2006
}

\author{
Rafael Teixeira ${ }^{1}$ \\ Daniel Pacheco Lacerda ${ }^{2}$
}

\begin{abstract}
Resumo: A crescente importância da cadeia de suprimentos tem levado pesquisadores a desenvolverem um volume significativo de trabalhos sobre o tema. Ao mesmo tempo, devido ao seu recente surgimento, esse fenômeno organizacional é complexo e engloba inúmeras variáveis, tornando igualmente complexo o processo de escolha do objeto de pesquisa por pesquisadores e estudantes. Por essa razão, esse artigo tem o objetivo de proporcionar uma análise dos principais tópicos estudados em 173 artigos sobre gestão da cadeia de suprimentos publicados nos principais periódicos acadêmicos internacionais entre 2004 e 2006. Os resultados mostram que os artigos focam, principalmente, a coordenação e a troca de informações entre empresas, a configuração da produção, o design e o desempenho da cadeia de suprimentos. Além disso, também são destacadas as características de alguns dos principais periódicos internacionais. Diversos tópicos, ainda, precisam ser estudados até que a gestão da cadeia de suprimentos se solidifique como disciplina.
\end{abstract}

Palavras-chave: Revisão de literatura. Gestão da cadeia de suprimentos. Artigos. Pesquisa. Periódicos acadêmicos.

\begin{abstract}
In recent years, supply chain management has received greater attention, hence leading researchers to develop many papers on this topic. Due to its recent development, this organizational phenomenon is complex and has many variables, making the process of choosing a research topic also complex for researchers and students. Thus, this paper aims to provide an analysis of the major topics studied in over 173 papers published about supply chain management in the most important international production and operation management journals between 2004 and 2006. The results show that the major topics are coordination, information sharing between companies, production decisions, and supply chain design and performance. Many topics should also be the focus of future studies until the supply chain management becomes a discipline.
\end{abstract}

Keywords: Supply chain management. Papers. Research. Journals. Topics.

\section{Introdução}

A gestão da cadeia de suprimentos vem se tornando uma área de crescente importância para as organizações nas últimas décadas em função de diversos fatores. Primeiro, as organizações estão focando as suas competências centrais e repassando aos fornecedores o desenvolvimento e suprimento de atividades e itens que suportam às suas atividades principais. Segundo, a globalização e internacionalização dos mercados requer que as organizações utilizem outras organizações para atender às demandas em diferentes países (STOREY et al., 2006) ou mesmo diferentes localidades dentro de um mesmo país. Terceiro, a própria segmentação do mercado (STOREY et al., 2006) exige que as organizações utilizem outros parceiros para ajudar a segmentar seus produtos, distribuição, vendas, entre outras atividades. Por fim, a complexidade gerencial das organizações tende a ser reduzida com a alocação de certas atividades para outras empresas.

\footnotetext{
${ }^{1}$ Department of Management, College of Business and Behavioral Science, 101, Sirrine Hall, Clemson University, Clemson, SC, 29634, E-mail: rafaelt@clemson.edu

${ }^{2}$ Programa de Pós-Graduação em Engenharia de Produção e Sistemas - PPGEPS/UNISINOS, Universidade do Vale do Rio dos Sinos - UNISINOS, Av. Unisinos, 950, CEP 93.022-000, São Leopoldo - RS, Brasil, E-mail: dlacerda@ unisinos.br
}

Recebido em 19/3/2009 — Aceito em 26/1/2010

Suporte financeiro: CAPES. 
A pesquisa acadêmica sobre gestão da cadeia de suprimentos também vem avançando crescentemente nas últimas décadas com amplos e diversificados estudos sobre o tema (BURGES; SINGH; KOROGLU, 2006). Estudos sobre os antecedentes necessários para a gestão da cadeia de suprimentos como, por exemplo, confiança e relacionamento (WU; CHOI, 2005; IRELAND; WEBB, 2006; McCARTER; NORTHCRAFT, 2006; GATTIKER; HUANG; SCHWARZ, 2007) e sobre as atividades organizacionais após as relações entre empresas já estarem desenvolvidas, tais como a troca de informações (BARRATT; OKE, 2006; CHU; LEE, 2006), a coordenação e cooperação entre empresas (SOUZA et al., 2004; DUDEK; STADLER, 2005; YUE et al., 2006) e a configuração da rede de fornecedores e parceiros (NAGURNEY et al., 2005; SIVADASAN et al., 2006) são alguns exemplos de trabalhos desenvolvidos. Outro conjunto de pesquisas é direcionado para os resultados da gestão da cadeia de suprimentos. Esses estudos buscam explorar as consequências das relações entre fornecedores e compradores ao longo da cadeia, como o efeito chicote (bullwhip effect) (MIYAOKA; HAUSMAN, 2004; SHEU, 2005), o desempenho (MARQUEZ; BIANCHI; GUPTA, 2004; RAY; LI; SONG, 2005; HULT et al., 2006) e a sustentabilidade da cadeia em si (LINTON; KLASSEN; JAYARAMAN, 2007).

Apesar do avanço prático e acadêmico, a gestão da cadeia de suprimentos ainda não se solidificou enquanto teoria ou mesmo disciplina (HARLAND et al., 2006; STOREY et al., 2006). Como uma área relativamente jovem, a gestão da cadeia de suprimentos necessita de medidas e construtos, definições e perspectiva teórica robusta (BURGES; SINGH; KOROGLU, 2006). Burges, Singh e Koroglu (2006) ainda utilizam diversas teorias para explicar o tema. Por exemplo, Tan (2001) tenta unir uma abordagem de compras e outra de logística para definir a gestão da cadeia de suprimentos, enquanto Chen e Paulraj (2004) utilizam uma perspectiva teórica de compras para justificar seu modelo. Outros autores tentam criar e estabelecer construtos, modelos e medidas para, por exemplo, as práticas na área (LI et al., 2005) e os antecedentes da agilidade na cadeia de suprimentos (SWAFFORD; GHOSH; MURTHY, 2006). De fato, áreas das ciências sociais têm tentado entender $\mathrm{e}$ explicar o fenômeno, embora a área de produção e operações estejam mais voltadas para o tema (BURGES; SINGH; KOROGLU, 2006).

Para solidificar-se como teoria e disciplina, a área requer, portanto, mais evidências, conclusões e debates (HARLAND et al., 2006). Diante de um tema complexo, relativamente amplo e ainda recente, o processo de escolha do objeto de pesquisa pelos pesquisadores e estudantes torna-se fundamental para que evidências empíricas ajudem o desenvolvimento da área. Apesar da contribuição e direcionamento proporcionados por artigos sobre o tema, não há um trabalho que sumarize os principais temas pesquisados e aponte lacunas a serem preenchidas por novas pesquisas.

O objetivo deste artigo é apresentar uma perspectiva sobre os estudos feitos e os principais tópicos pesquisados sobre gestão da cadeia de suprimentos publicados nos principais periódicos acadêmicos internacionais da área de gestão de produção e operações entre os anos de 2004 e 2006. Para isso, apresenta-se um modelo de gestão da cadeia de suprimentos que serve de referência para este estudo. Duas dimensões foram utilizadas para classificar os artigos analisados: característica do periódico acadêmico (qualidade-relevância) e metodologia empregada (empiricismo-modelagem). Como consequência, uma das contribuições deste trabalho é apresentar tópicos de pesquisa na área que ainda precisam ser explorados, servindo de base para futuras pesquisas. Outra contribuição é sumarizar brevemente os principais resultados encontrados nas pesquisas desenvolvidas. Uma última contribuição é prover ao leitor uma análise de como os artigos desenvolvidos por acadêmicos podem ser posicionados em função das características dos periódicos acadêmicos internacionais.

$\mathrm{O}$ artigo está divido em cinco partes: primeiro, uma breve revisão teórica é apresentada para sintetizar os principais tópicos de pesquisa na área; segundo, a metodologia utilizada para a coleta e análise dos dados é discutida; terceiro, os resultados são apresentados; quarto, uma seção discute os principais resultados; por fim, as conclusões e limitações são abordadas.

\section{Referencial teórico}

A gestão da cadeia de suprimentos tem recebido crescente atenção como objeto de pesquisa, levando pesquisadores a diferentes definições sobre o tema. Algumas dessas definições deixam o conceito amplo (HARLAND; LAMMING; COUSINS, 1999) ou trazem o viés de uma teoria que influencia determinado autor (TAN, 2001). De fato, cada definição varia dependendo da perspectiva teórica utilizada para abordar o objeto. Analisando algumas definições de gestão da cadeia de suprimentos, ainda se notam variações que tornam o fenômeno incompleto. Por exemplo, a definição da gestão da cadeia de suprimentos proposta por Chen e Paulraj (2004) valoriza os elementos que levam as empresas a fazerem e manterem a gestão da relação com seus fornecedores, mas não inclui resultados e consequências dessas práticas. Li et al. (2005) restringem o conceito às atividades essenciais para sua gestão, mas falham ao não incluir possíveis antecedentes e consequentes.

$\mathrm{Na}$ tentativa de proporcionar uma definição ampla e completa sobre o tema, Mentzer et al. (2001) analisaram diversos estudos sobre a gestão da cadeia 
de suprimentos. De acordo com os autores, gestão da cadeia de suprimentos é definida como uma

“coordenação sistemática e estratégica das funções gerenciais tradicionais e das táticas entre elas dentro de uma empresa e, entre empresas, dentro da cadeia de suprimentos, para melhoria do desempenho de longo prazo das empresas separadamente e da cadeia como um todo" (MENTZER et al., 2001, p.18).

O modelo teórico proposto por Mentzer et al. (2001) (conforme Figura 1) sintetiza as principais variáveis que compõem a cadeia de suprimentos ao mesmo tempo que delimita-as sucintamente.

Esse modelo teórico aborda o tema de um ponto de vista amplo, englobando antecedentes, atividades centrais e consequentes. Esse tipo de modelo facilita a compreensão das variáveis e de suas relações, embora simplifique o fenômeno. Outra vantagem desse modelo é que evita pressupostos com relação a outras perspectivas teóricas tais como, por exemplo, compras (TAN, 2001; CHEN; PAULRAJ, 2004) ou logística (TAN, 2001), proporcionando maior isenção teórica. Dessa forma, o modelo de Mentzer et al. (2001) permite a conjunção e análise de um maior número de variáveis.

Os antecedentes, juntamente com a orientação da empresa, formam o conjunto de tópicos que são necessários à formação de uma cadeia de suprimentos. As empresas têm que estar dispostas a confiar e comprometer-se com seus fornecedores e clientes, tornando-se interdependente, com a visão e os processos-chave voltados para a cadeia de suprimentos (LI et al., 2005). Para que seja factível, esses itens requerem suporte do líder e da alta direção da organização (CHEN; PAULRAJ, 2004; BURGES; SINGH; KOROGLU, 2006). Esse conjunto de variáveis forma a visão sistêmica e estratégica que as organizações têm que ter para enxergar a cadeia de suprimentos como um todo. A visão sistêmica permite que as organizações compreendam os efeitos de suas ações para todas as outras organizações na cadeia até o cliente final, permitindo-se, assim, valerem-se estrategicamente de sua posição (TAN, 2001).

As atividades de gestão da cadeia de suprimentos formam o segundo bloco de variáveis do modelo. De maneira resumida, pode-se dizer que, para existir gestão na cadeia de suprimentos, é necessário três ou mais empresas que compartilhem da mesma visão estratégica e dos mesmos objetivos, riscos e resultados (MENTZER et al., 2001; TAN, 2001). Esse compartilhamento pressupõe que as empresas cooperem e busquem a coordenação de áreas específicas dentro de cada empresa e entre as empresas da cadeia, trocando informações e integrando processos-chave, permitindo que a informação e o fluxo de materiais ocorram de maneira eficiente (BURGES; SINGH;
KOROGLU, 2006; STOREY et al., 2006). Embora não esteja no modelo de Mentzer et al. (2001), o intenso e complexo fluxo de informações na cadeia exige que as empresas disponham de suficientes recursos e competências em tecnologia da informação (CHEN; PAULRAJ, 2004). Alterações e adaptações desse porte requerem que as empresas mantenham o relacionamento por um longo período de maneira que o retorno para tais investimentos seja obtido (CHEN; PAULRAJ, 2004; BURGES; SINGH; KOROGLU, 2006). Além disso, como cada empresa dispõe de uma rede de relacionamentos específica para sua operação, o design da cadeia de suprimentos é fundamental para maximizar os ganhos de cada empresa e da cadeia em si.

As consequências da gestão da cadeia de suprimentos formam o último grupo de variáveis do modelo, relacionadas aos produtos da gestão (BURGES; SINGH; KOROGLU, 2006). Por exemplo, espera-se que o envolvimento de diversas empresas na gestão da cadeia reduza seus custos, melhore seus desempenhos e, por consequência, proporcione maior valor e satisfação aos seus clientes (MENTZER et al., 2001; TAN, 2001). Embora não esteja no modelo original, a gestão da cadeia de suprimentos também tem efeitos para a sua auto-sustentabilidade e para o efeito chicote (bulwhipp effct), que será detalhado na seção Resultados e Análises. Além disso, a interrupção de suprimentos afeta diretamente as empresas da cadeia, provocando prejuízos às suas operações e ao valor de suas ações no mercado de capitais (HENDRICKS; SINGHAL, 2005a; 2005b).

\section{Procedimento metodológico}

Primeiramente foram selecionados periódicos acadêmicos da área de produção e operações que servissem de base para a coleta dos artigos. Priorizou-se a área de produção e operações, pois é a área que possui um volume maior de pesquisas sobre esse

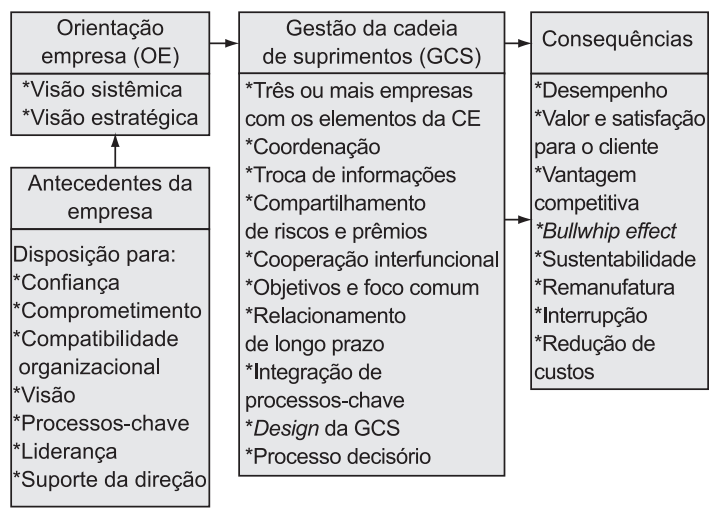

Figura 1. Modelo teórico de antecedentes e consequentes da gestão da cadeia de suprimentos. Adaptado de Mentzer et al. (2001). 
fenômeno (BURGES; SINGH; KOROGLU, 2006). Os periódicos acadêmicos foram selecionados baseados nos resultados da pesquisa de Vasilis et al. (2007). Esse trabalho foi utilizado como referência pelos seguintes motivos: i) é a mais recente pesquisa sobre o tema; ii) analisa resultados de pesquisas anteriores; iii) proporciona novos insigths sobre o tópico; e iv) considera diferenças entre trabalhos empíricos e de trabalhos de modelagem.

Vasilis et al. (2007) entrevistaram acadêmicos de diversos continentes para avaliar suas percepções em relação aos periódicos acadêmicos existentes na área de produção e operações. De acordo com os resultados, os periódicos podem ser classificados em duas dimensões: relevância e qualidade. A dimensão relevância refere-se à importância do tema estudado para os problemas gerenciais. Um trabalho voltado para a solução de problemas gerenciais é um trabalho relevante. A dimensão qualidade refere-se à importância dos problemas analisados para a teoria e o rigor metodológico empregado nos artigos.

Baseado nos resultados encontrados por Vasilis et al. (2007), os seguintes periódicos acadêmicos foram selecionados para servir de base para a coleta de artigos: Management Science (MS), Journal of Operations Management (JOM), Production and Operations Management (POM), Decision Sciences (DS), Manufacturing and Service Operations Management (MSOM), International Journal of Operations and Production Management (IJOPM) e European Journal of Operational Research (EJOR).

Para além dos resultados encontrados por Vasilis et al. (2007), este artigo procurou articular a contribuição de Vasilis et al. (2007) com o modelo proposto por Mentzer et al. (2001) conforme já exposto na Figura 1. Assim, para os tópicos considerados relevantes, foram posicionados os artigos em função do modelo apresentado por Vasilis et al. (2007). Esta é, portanto, outra contribuição da presente pesquisa que procurou articular diferentes modelos para o enquadramento da produção científica que é o objeto desta pesquisa. Além disso, para fins ilustrativos, foi incluído o periódico Gestão \& Produção com o objetivo de fornecer indícios, de certa maneira, da pesquisa nacional no cenário internacional.

Foram analisados os artigos publicados nos periódicos citados acima durante os anos de 2004, 2005 e 2006. A seleção de artigos foi feita a partir da análise das palavras-chave e abstracts. Dois estudantes de doutorado da área de produção e operações fizeram a seleção dos artigos e um professor, doutor na área de operações e produção, fez a avaliação final com base nos abstracts. A amostra final foi composta de um total de 173 artigos.

Os artigos foram analisados e classificados de acordo com os tópicos de pesquisa. Por exemplo, artigos sobre coordenação na cadeia de suprimentos foram agrupados, artigos sobre interrupção na cadeia de suprimentos formaram outro grupo e assim por diante. Nenhum artigo foi classificado em mais de um grupo, embora houvesse artigos que abordassem mais de um tópico. Por fim, os tópicos são classificados em antecedentes, atividades centrais da gestão da cadeia de suprimentos e suas consequências, seguindo o modelo teórico (Figura 1) de Mentzer et al. (2001). Isso permite a análise dos artigos dentro de tópicos mais amplos.

Além disso, os artigos também foram analisados de acordo com a metodologia empregada, pois existem trabalhos que utilizam uma abordagem empírica e outros que utilizam uma abordagem de modelagem. Essa distinção contribui para o entendimento dos artigos analisados.

Os artigos foram classificados em duas dimensões: 1) característica do periódico: relevância-qualidade; $\mathrm{e}$ 2) metodologia empregada: empiricismo-modelagem. A dimensão característica do periódico ficou em um eixo com relevância e qualidade compondo um continuum. Entretanto, a utilização do continuum não significa a mútua exclusão de um periódico que se encontra em uma determinada parte do eixo. Esse procedimento foi utilizado apenas para viabilizar a representação gráfica dos resultados para facilitar o entendimento pelo leitor. A dimensão da metodologia empregada ficou em outro eixo com modelagem e empiricismo formando outro continuит. As diversas metodologias, tais como, por exemplo, estudo de caso ou modelagem determinística, entre outras, ficaram dentro desse continuum com trabalhos quantitativos representados no extremo dos trabalhos empíricos e a modelagem estocástica, por exemplo,

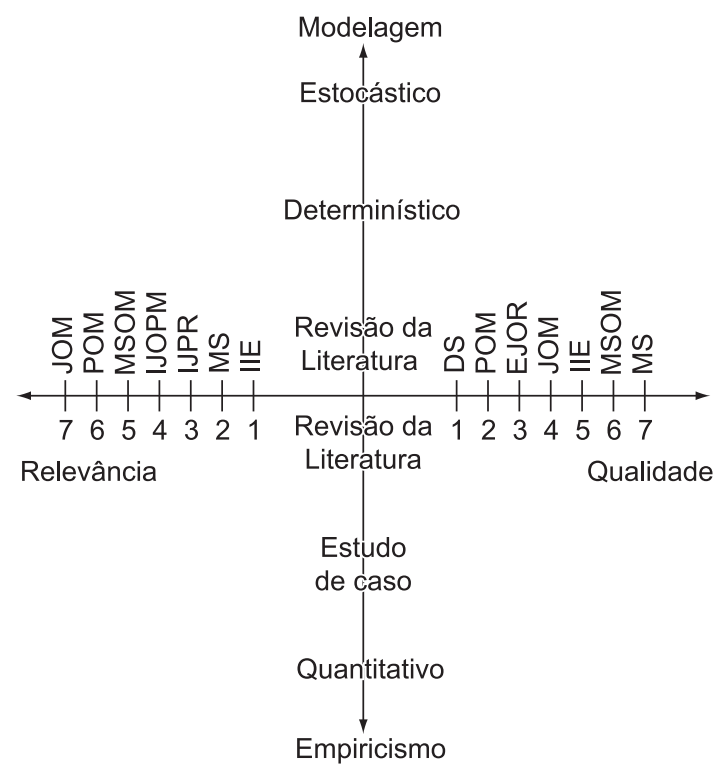

Figura 2. Gráfico da característica do periódico e a metodologia empregada. Fonte: Autores (2010). 
representada no extremo dos trabalhos de modelagem. A Figura 2 ilustra as duas dimensões e busca auxiliar na compreensão do leitor sobre a abordagem empregada para classificação dos artigos.

Em um extremo, os trabalhos empíricos (empiricismo) são aqueles que lidam com a realidade concreta como elemento para fundamentação de suas conclusões. Por um lado, as hipóteses (estudos quantitativos) ou proposições (estudos qualitativos) sejam deduzidas elas são testadas com os dados reais (VASILIS et al., 2007). Por outro lado, ainda dentro do empiricismo, estão os estudos indutivos que procuram, a partir dos dados, inferir fatores, conclusões e outros resultados.

Em outro extremo, estão as pesquisas que se utilizam da construção de modelos (modelagem) para compreender e, em alguns casos tentar, predizer a realidade. Os modelos podem ser matemáticos, diagramas, simulação computacional (eventos discretos, sistemas dinâmicos, por exemplo) dentre outras abordagens (VASILIS et al., 2007). Uma característica dos modelos é que podem ser determinísticos ou estocásticos. Os modelos determinísticos não consideram a variabilidade. Por sua vez, os modelos estocásticos consideram a aleatoriedade pelo uso de distribuições probabilísticas (PIDD, 1998).

Os periódicos acadêmicos foram ordenados no eixo de acordo com as suas respectivas posições a partir dos resultados de Vasilis et al. (2007). Por exemplo, de acordo com os resultados encontrados, o Journal of Operations Management (JOM) é o mais relevante enquanto que o Management Science (MS) é o que tem maior qualidade.

Esse gráfico serve de base para a classificação dos artigos analisados e posiciona-os dentro de cada eixo, permitindo ao leitor uma visão geral de algumas características dos artigos sobre gestão da cadeia de suprimentos. Todo o processo de análise e classificação dos artigos foi feita, novamente, por dois estudantes de doutorado da área de produção e operações e validadas por um professor da áreas.

\section{Resultados e análises}

A primeira análise levou em consideração os tópicos pesquisados em gestão da cadeia de suprimentos. Os artigos foram classificados levando em consideração os tópicos do modelo teórico da Figura 1. A Tabela 1 apresenta a quantidade de artigos para cada tópico, a classificação dos tópicos e sua frequência relativa.

Os tópicos mais pesquisados são os relacionados com as atividades da gestão da cadeia de suprimentos (60\%) e englobam coordenação entre fornecedores e compradores $(12 \%)$, troca de informações entre as empresas da cadeia (9\%), configuração da produção (8\%) e design da cadeia (8\%). Os tópicos relacionados às consequências da gestão da cadeia de suprimentos representam $24 \%$ de todos os artigos analisados e o impacto da gestão da cadeia para o desempenho das empresas é o tópico mais pesquisado. Os antecedentes representam o menor grupo de artigos, somando $8 \%$ do total. Por fim, um total de 11 artigos (8\%) não foi utilizado na análise por não se enquadrarem nos objetivos propostos neste trabalho. Esses artigos tratavam de aplicação prática de modelagem à cadeia de suprimentos, metodologias aplicadas à gestão da cadeia de suprimentos ou não eram de fato artigos sobre o tema.

Alguns tópicos não constam no modelo original de Mentzer et al. (2001) e foram incluídos, pois são importantes para a gestão da cadeia de suprimentos, tais como configuração da produção, uso de contratos e o efeito chicote. Para não excluí-los e por consequência, distorcer os resultados, esses tópicos foram incluídos nas análises.

Por fim, é importante destacar que nem todos os artigos puderam ser citados nas análises feitas a seguir porque a grande quantidade de tópicos e artigos impossibilita a análises de todos. Os 150 artigos internacionais analisados neste trabalho encontram-se no Anexo I. Por sua vez, foram analisados 23 artigos sobre gestão da cadeia de suprimentos do periódico Gestão \& Produção e encontram-se no Anexo II.

\subsection{Análise dos artigos sobre antecedentes e orientação da empresa}

Os antecedentes, juntamente com a orientação da empresa, formam o conjunto de tópicos que são necessários à composição de uma cadeia de suprimentos. Logo, esses tópicos são fundamentais para compreender a própria existência da cadeia de suprimentos. A Figura 3 mostra a classificação dos artigos nas dimensões característica do periódico versus a metodologia empregada. Utilizaram-se pontos de densidade para ilustrar graficamente a quantidade de artigos desenvolvidos em cada quadrante do gráfico. A maioria dos artigos (66\%) foram estudos sobre confiança e relacionamentos entre empresas com o objetivo de avaliar as repercussões para a cadeia de suprimentos.

\section{a) Artigos sobre confiança}

Dentre os artigos sobre confiança, dois foram revisões teóricas que exploraram a cadeia de suprimentos como um dilema social (McCARTER; NORTHCRAFT, 2006) e trouxeram outras perspectivas teóricas para explicar confiança e poder nas cadeias de suprimentos (IRELAND; WEBB, 2006), enquanto outros dois artigos foram pesquisas empíricas quantitativas para entender os efeitos da informação para a confiança (GATTIKER; HUANG; SCHWARZ, 2007) e os efeitos da confiança para o desempenho das empresas (JOHNSTON et al., 2004). Os principais resultados mostram a importância da informação para a confiança e a importância da confiança para o desempenho. 
Tabela 1. Classificação dos artigos em tópicos de pesquisa.

\begin{tabular}{|c|c|c|c|c|c|}
\hline Tópico & $\begin{array}{l}\text { Número de } \\
\text { artigos }\end{array}$ & $\begin{array}{l}\text { Frequência } \\
(\%)\end{array}$ & $\begin{array}{c}\text { Classificação } \\
\text { do tópico }\end{array}$ & $\begin{array}{l}\text { Número de } \\
\text { artigos }\end{array}$ & $\begin{array}{c}\text { Frequência } \\
(\%)\end{array}$ \\
\hline Confiança & 4 & 3 & \multirow{6}{*}{$\begin{array}{c}\text { Antecedentes e } \\
\text { orientação da empresa }\end{array}$} & \multirow[t]{6}{*}{12} & \multirow[t]{6}{*}{8} \\
\hline Relacionamento & 4 & 3 & & & \\
\hline Liderança & 1 & 1 & & & \\
\hline Visão estratégica & 1 & 1 & & & \\
\hline Perfil dos gerentes & 1 & 1 & & & \\
\hline Implementação da GCS & 1 & 1 & & & \\
\hline Coordenação & 18 & 12 & \multirow{12}{*}{$\begin{array}{l}\text { Atividades da gestão da } \\
\text { cadeia de } \\
\text { suprimentos }\end{array}$} & \multirow[t]{12}{*}{91} & \multirow[t]{12}{*}{60} \\
\hline Troca de informações & 14 & 9 & & & \\
\hline Configuração da produção & 12 & 8 & & & \\
\hline Design da CS & 12 & 8 & & & \\
\hline Integração de processos chave & 9 & 6 & & & \\
\hline Compartilhamento de riscos & 6 & 4 & & & \\
\hline Inventário & 4 & 3 & & & \\
\hline Agilidade na GCS & 4 & 3 & & & \\
\hline Uso da tecnologia da informação & 4 & 3 & & & \\
\hline Relacionamento de longo prazo & 3 & 2 & & & \\
\hline Planejamento de produção & 3 & 2 & & & \\
\hline Contratos & 2 & 1 & & & \\
\hline Desempenho & 16 & 11 & \multirow[t]{6}{*}{ Consequências } & \multirow[t]{6}{*}{35} & \multirow[t]{6}{*}{24} \\
\hline Efeito chicote & 6 & 4 & & & \\
\hline Interrupções na cadeia & 5 & 3 & & & \\
\hline Sustentabilidade & 4 & 3 & & & \\
\hline Remanufatura & 3 & 2 & & & \\
\hline Redução de custos & 1 & 1 & & & \\
\hline Aplicação de modelagem & 5 & 3 & \multirow{4}{*}{$\begin{array}{c}\text { Não são artigos } \\
\text { de GCS }\end{array}$} & \multirow[t]{3}{*}{12} & \multirow[t]{3}{*}{8} \\
\hline Metedologia & 5 & 1 & & & \\
\hline Não eram artigos de GCS & 2 & 1 & & & \\
\hline Total & 150 & 100 & & 150 & 100 \\
\hline
\end{tabular}

Fonte: Autores (2010).

\section{b) Artigos sobre relacionamento}

Os artigos sobre relacionamento entre empresas, um múltiplo estudo de caso foi conduzido sobre o relacionamento entre fornecedores de um mesmo cliente (WU; CHOI, 2005). Outros três artigos foram pesquisas quantitativas empíricas para identificar como as características das empresas podem influenciar seus comportamentos no relacionamento (VAART; DONK, 2008), a importância das relações formais e informais (COUSINS et al., 2006) e o impacto dos sistemas interorganizacionais para as relações entre comprador-vendedor (SAEED; MALHOTRA; GROVER, 2005).

\section{c) Discussão dos artigos sobre antecedentes e orientação da empresa}

Esses resultados, juntamente com os resultados da Figura 3, permitem inferir algumas conclusões.
Primeiro, os poucos artigos desenvolvidos sobre os antecedentes da gestão da cadeia de suprimentos foram quase, exclusivamente, empíricos. Do total de 12 artigos, 7 foram trabalhos empíricos, sendo que, desses, 3 foram estudos de caso e 4 , trabalhos quantitativos. A revisão de literatura foi o foco de 4 artigos e, somente, 1 artigo foi realizado utilizando modelagem de dados. Esses resultados parecem mostrar que os antecedentes da gestão da cadeia de suprimentos ainda não foram bem explorados. Pelo contrário, a quantidade de artigos sobre revisão da literatura $(33 \%)$ parece mostrar que as pesquisas ainda estão fazendo levantamentos bibliográficos para compreender as variáveis que antecedem a formação das cadeias de suprimentos.

Segundo, os periódicos acadêmicos JOM e IJOPM foram responsáveis pela publicação de 10 dos 12 artigos sobre os antecedentes da gestão da cadeia de suprimentos e 6 dos 8 artigos utilizando métodos 


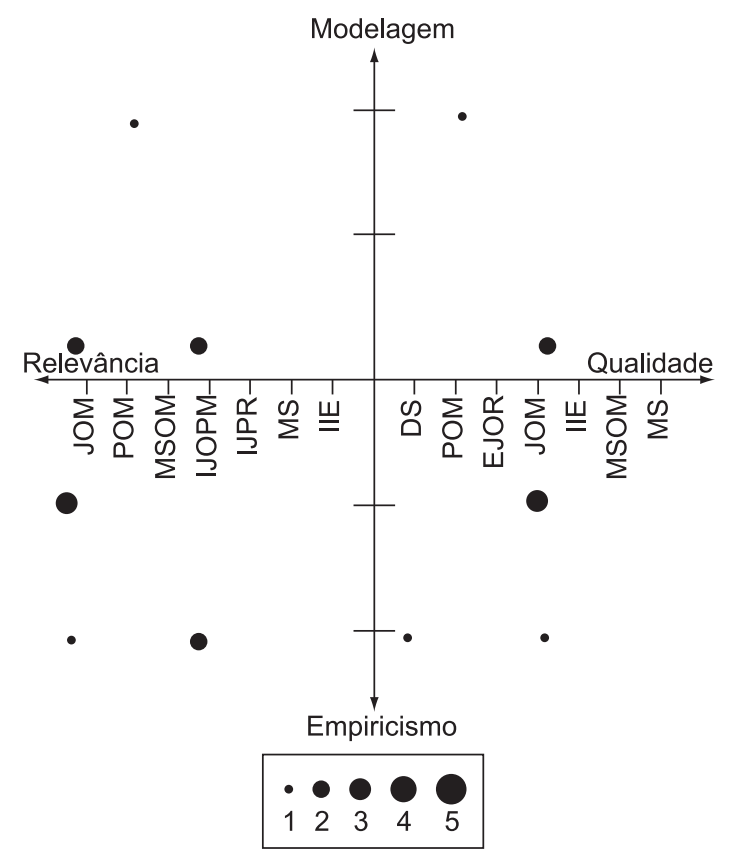

Figura 3. Classificação dos artigos sobre antecedentes e orientação da empresa Fonte: Autores (2010).

empíricos. Esse resultado mostra o caráter empírico desses dois periódicos e suas preocupações sobre os antecedentes da cadeia de suprimentos. Pesquisadores e acadêmicos que realizam trabalhos empíricos sobre os antecedentes da cadeia de suprimentos têm, potencialmente, maiores chances de publicação nesses periódicos internacionais.

Em terceiro lugar, poucos trabalhos foram desenvolvidos sobre as variáveis que antecedem a formação das cadeias de suprimentos, conforme já havia sido evidenciado na Tabela 1. A maioria desses artigos (66\%) foi sobre confiança e relacionamento entre as empresas na cadeia de suprimentos. Esse resultado mostra uma preocupação com os princípios básicos de formação de parceria entre as empresas, um dos fundamentos que sustentam a existência da cadeia de suprimentos. Entretanto, existem lacunas a serem preenchidas, principalmente na condução de trabalhos que busquem explorar os diversos tópicos que levam à formação das cadeias de suprimentos. Por exemplo, o papel do líder e da alta direção pode ser vital para que qualquer projeto organizacional tenha efeito para a organização. Um projeto sem apoio dos líderes da empresa tende a resultados igualmente fracos.

Dessa forma, acadêmicos que estudem a percepção de acionistas, presidentes e da alta direção em relação à gestão da cadeia de suprimentos permitirão o entendimento de variáveis que podem determinar a formação, consecução e sucesso das cadeias de suprimentos. A lista de variáveis do modelo de Mintzer et al. (2001) é uma fonte de ideias para futuros artigos. Pesquisadores e acadêmicos podem valer-se desse, e de outros modelos, para direcionar seus esforços de pesquisa para os tópicos que estejam carentes de pesquisa.

\subsection{Análise dos artigos sobre as atividades na gestão da cadeia de suprimentos}

As atividades na gestão da cadeia de suprimentos compõem o maior conjunto de tópicos. Esse resultado deve-se à importância dessas atividades para manutenção e evolução da cadeia. Talvez, por esse motivo, esses tópicos sejam os mais pesquisados na amostra analisada (conforme Tabela 1).

A Figura 4 mostra a classificação desses artigos nas dimensões: i) característica do periódico $v s$. ii) metodologia empregada. Utilizaram-se pontos de densidade para ilustrar graficamente a quantidade de artigos desenvolvidos em cada quadrante do gráfico para proporcionar uma ideia do volume de artigos publicados em cada periódico e o método empregado.

Mais de $60 \%$ dos artigos foram baseados em estudos que empregaram a metodologia de modelagem, como pode ser percebido pela Figura 4. A maioria dos artigos (61\%) abordou os seguintes tópicos: i) coordenação entre as empresas; ii) troca de informações entre empresas; iii) configuração da produção entre duas ou mais empresas; e iv) design da cadeia de suprimentos.

\section{a) Artigos sobre coordenação}

Os artigos sobre coordenação entre as empresas foram baseados quase que exclusivamente em modelagem e focaram, principalmente, o planejamento conjunto da produção (POUNDARIKAPURAM; VEERAMANI, 2004; DUDEK; STADLER, 2005; DAWANDE et al., 2006), das vendas (SOUZA et al., 2004; BERNSTEIN; CHEN; FEDERGRUEN, 2006) e da propaganda (YUE et al., 2006). Em geral, os resultados destacam a redução de custos e melhoria do desempenho das empresas como os principais benefícios para o comprador e o fornecedor que colaboram entre si.

Outro conjunto de artigos sobre esse tópico volta-se para a relação conflituosa que pode existir entre compradores e fornecedores, sob diversas características, tais como: comprador e fornecedor competindo pelo mesmo cliente (BOYACI, 2005), competição entre cadeias de suprimentos (BOYACI; GALLEGO, 2004) e cadeias de suprimentos descentralizadas (WANG; GUO; EFSTATHIOU, 2004; BERNSTEIN; FEDERGRUEN, 2005). Os resultados desses trabalhos são divergentes, não conclusivos e, dentro de um cenário de competição, levantam dúvidas 


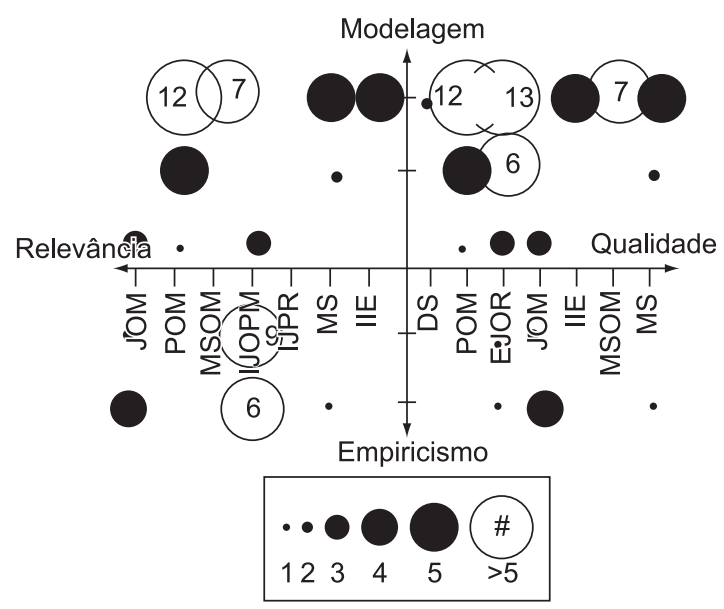

Figura 4. Classificação dos artigos sobre atividades de gestão da cadeia de suprimentos. Fonte: Autores (2010).

quanto aos benefícios da coordenação e colaboração entre as empresas.

\section{b) Artigos sobre troca de informações entre empresas}

A troca de informações entre empresas tem sido outro tópico de destaque no conjunto de variáveis que compõem as atividades da cadeia de suprimentos. $\mathrm{O}$ impacto no desempenho das empresas (SANDERS, 2007; ZHOU; BENTON Jr., 2007) e o uso da internet, que facilitam e aumentam a integração entre comprador e vendedor (CROOM, 2005), talvez sejam duas razões para tal importância. A vantagem competitiva obtida com o gerenciamento de recursos para a troca de informação entre as empresas (BARRATT; OKE, 2007) e os benefícios, como redução de custos (GÜLLÜ et al., 2005), também são razões que tornam o tema importante.

Alguns autores apresentam modelos que avaliam o uso da tecnologia da informação para a integração entre empresas (THEMISTOCLEOUS; IRANI; LOVE, 2004; CAGLIANO; CANIATO; SPINA, 2005) e princípios preliminares que as empresas podem seguir antes da efetiva troca de informações (LOCH; TERWIESCH, 2005). Outros apontam a falta de liderança e de estratégia conjunta na cadeia de suprimentos (HARLAND et al., 2007) e o custo elevado da troca de informações (CHU; LEE, 2006) como algumas das principais barreiras para sua adoção.

\section{c) Artigos sobre configuração da produção}

Esses artigos abordam os efeitos de diferentes variáveis da cadeia de suprimentos sobre a configuração da produção. Por exemplo, Rao, Swaminathan e Zhang (2005) estudaram como uma empresa pode integrar decisões de produção e demanda garantindo um lead time uniforme aos clientes. Ray, Gerchak e Jewkes (2004) analisaram os impactos da redução no lead time entre comprador e vendedor verificando uma redução nos custos de produção. Já Hazra, Mahadevan e Seshadri (2004) estudaram a decisão de preços e capacidade de produção quando os fornecedores operam em mercados eletrônicos (internet) e estimaram o nível ótimo de fornecedores.

Uma série de outros artigos enfocou, dentre outros temas, a utilização de práticas de just-in-time em uma cadeia (WANG; SARKER, 2006), as políticas ótimas de produção, distribuição (AHN; KAMINSKY, 2005), programação da produção (SELVARAJAH; STEINER, 2006) e precificação (CATTANI et al., 2006) entre comprador e vendedor. Esses artigos utilizaram a metodologia de modelagem e seus principais resultados foram algoritmos e heurísticas para a solução dos problemas dentro de contextos de operações específicos.

Por fim, outro conjunto de artigos focou o problema dos inventários na cadeia de suprimentos. Sobel e Fil (2004) modelou a taxa de completamento de estoques e mostrou que cadeia de suprimentos menores tem maiores taxas de completamento de estoque, enquanto Simchi-Levi e Zhao (2005) e Daniel e Rajendran (2006) apresentaram algoritmos para, respectivamente, o problema da posição dos estoques de segurança e para os níveis de estoque em cadeias com diferentes configurações.

\section{d) Artigos sobre design da cadeia de suprimentos}

Os artigos sobre esse tópico podem ser subdividos em dois grupos: artigos que se basearam em estudos de caso e revisão de literatura e os que utilizaram a metodologia de modelagem. Os artigos do primeiro grupo analisaram o impacto da internet para a re-configuração da indústria fonográfica (GRAHAM et al., 2004), a influência da posição da empresa-foco no design de uma rede de suprimentos (CHOI; KRAUSE, 2006) e o impacto das redes de serviços de manutenção remota (PERSONA et al., 2007), da arquitetura de produtos e processos (FIXSON, 2005) e da personalização de produtos (POULIN; MONTREUIL; MARTEL, 2006), para a configuração da cadeia de suprimentos. Os principais resultados mostram que a tecnologia da informação e o número de fornecedores em uma rede de suprimentos têm importantes implicações para o design da cadeia.

O segundo grupo de estudos utilizou uma abordagem de modelagem para estudar o design da cadeia de suprimento. Os principais resultados foram 
algoritmos para solucionar, entre outros, os seguintes problemas: um modelo ótimo combinando a família de produtos e a cadeia de suprimentos (LAMOTHE; HADJ-HAMOU; ALDANONDO, 2006); um modelo de rede de suprimentos incluindo risco e incerteza (NAGURNEY et al., 2005); uma medida para avaliar complexidade operacional entre comprador e vendedor (SIVADASAN et al., 2006); e a ótima configuração de uma cadeia com canais diretos e indiretos (SEIFERT; THONEMANN; SEIKE, 2006).

\section{e) Discussão dos artigos sobre as atividades da cadeia de suprimentos}

As atividades da cadeia de suprimentos são o conjunto de variáveis com maior número de pesquisas realizadas. De acordo com os resultados da Tabela 1 , $60 \%$ dos artigos foram relativos ao tema. Além disso, a Figura 3 mostra que a maioria desses artigos (62\%) utilizou a metodologia de modelagem (57 do total de 91 artigos). Esses resultados mostram que os pesquisadores estão preocupados com o desenvolvimento das ações gerenciais que afetam diretamente o fenômeno. A ênfase em modelagem reflete uma preocupação em analisar os efeitos de diversas variáveis gerenciais para o desempenho direto ou indireto da cadeia.

Embora a ênfase dos artigos na metodologia de modelagem, em geral, há pesquisas realizadas utilizando todos os tipos de metodologia. A menor quantidade de pesquisas empíricas, sejam estudos de caso ou pesquisas quantitativas, mostram uma oportunidade para que pesquisadores desenvolvam pesquisas utilizando essa abordagem. A utilização das diversas metodologias é necessária para que os resultados encontrados sobre determinado tópico possam ser confirmados a partir de dados reais.

Há ainda diversas variáveis ainda não exploradas e que podem servir de base para futuros estudos. Por exemplo, cooperação interfuncional é um problema ainda existente nas empresas e que pode ter efeitos consideráveis para a gestão da cadeia de suprimentos que ainda não foram estudados. Pesquisadores podem estudar a colaboração de uma mesma área entre as empresas da cadeia. Levando em consideração a importância da coordenação e a troca de informações entre as empresas, como as áreas de tecnologia da informação de cada empresa da cadeia enxergam a cooperação entre elas com o objetivo de maximizar lucros ou reduzir custos? Como as áreas de produção, logística e compras podem interagir ao longo da cadeia para melhorar o desempenho de todos? Essas questões remetem a outras variáveis também pouco exploradas: a dos objetivos e focos comuns. Como as empresas estabelecem objetivos comuns? Existe uma empresa líder na cadeia que proporciona um direcionamento das outras empresas em direção ao mesmo foco? Quais as implicações para o relacionamento a longo prazo e design da cadeia, quando levam em consideração os objetivos e focos comuns?

\subsection{Análise dos artigos sobre as consequências da gestão da cadeia de suprimentos}

Os artigos publicados sobre as consequências da gestão da cadeia de suprimentos compõem o segundo maior conjunto de tópicos analisados. A principal razão para esse resultado talvez seja a mesma que leva muitos pesquisadores e gerentes a desenvolverem suas atividades diariamente: a busca de melhores resultados. Isto é, qual a razão para tanto esforço em pesquisa senão a melhoria contínua da pesquisa acadêmica e das atividades empresariais? Entretanto, também cabe uma crítica: se a busca de melhores resultados da gestão da cadeia de suprimentos (melhores consequências, usando o subtítulo aqui empregado) é o principal motivo pelo qual todos se esforçam, por que ainda há poucos trabalhos nessa categoria, quando em comparação com os trabalhos desenvolvidos sobre as atividades da gestão da cadeia de suprimentos? Essa constatação apresenta uma importante oportunidade para pesquisadores interessados em desenvolver trabalhos na área.

A Figura 5 apresenta a classificação desses artigos nas dimensões: i) característica do periódico vs ii) metodologia empregada. Utilizaram-se pontos de densidade para ilustrar graficamente a quantidade de

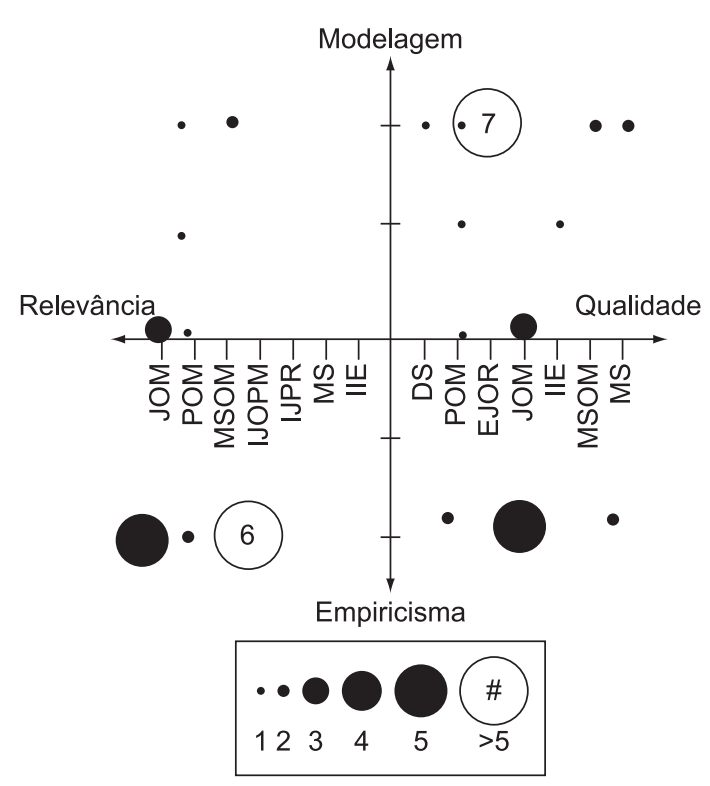

Figura 5. Classificação dos artigos sobre as consequências da gestão da cadeia de suprimentos. Fonte: Autores (2010). 
artigos desenvolvidos em cada quadrante do gráfico para ilustrar o volume de artigos publicados em cada periódico e o método empregado. Os artigos dentro dessa categoria representam $24 \%$ do total de artigos analisados, sendo que a maioria destes $(45 \%)$ enfoca o desempenho das empresas na cadeia de suprimentos ou o desempenho da própria cadeia de suprimentos e o efeito chicote $(17 \%)$.

\subsection{Análise dos periódicos acadêmicos internacionais da área de produção e operações}

As duas dimensões utilizadas para posicionar os artigos analisados permitem inferir sobre o perfil dos periódicos acadêmicos internacionais utilizados como base para esse estudo. Os resultados mostram a formação de dois conjuntos de periódicos: i) os periódicos que publicam trabalhos empíricos; e ii) os que publicam trabalhos baseados em modelagem. Esse resultado pode não ser uma novidade para pesquisadores experientes, mas pode auxiliar estudantes a identificar os periódicos acadêmicos que privilegiam determinada metodologia em detrimento de outras.

Os periódicos que publicam maior quantidade de trabalhos empíricos são: JOM, IJOPM e POM. Existem alguns periódicos, tais como DS e MS, que publicam resultados de trabalhos empíricos, mas em menor quantidade. Os periódicos que publicam maior quantidade de trabalhos baseados em modelagem são: EJOR, MSOM, MS e DS. Periódicos do primeiro grupo também publicam resultados de trabalhos baseados em modelagem, mas em menor quantidade.

Além disso, Vasilis et al. (2007) concluíram que tais grupos de periódicos podem ser classificados em relação a sua relevância e qualidade. $\mathrm{O}$ aspecto relevância diz respeito à importância do tópico estudado para a resolução de problemas gerenciais. Por um lado, o grupo de periódicos no eixo relevância (Figura 2) é mais propenso a publicar resultados de trabalhos que tenham repercussão para as práticas gerenciais. Por outro lado, o grupo de periódicos classificadas como de maior qualidade prima por maior rigor metodológico.

Os resultados dos artigos analisados mostram que os trabalhos sobre gestão da cadeia de suprimentos foram desenvolvidos utilizando mais uma abordagem de modelagem do que uma abordagem empírica. Por consequência, houve uma maior publicação dos artigos em periódicos acadêmicos, ditos de maior qualidade. Espera-se, portanto, que essa constatação encoraje pesquisadores e estudantes a desenvolverem pesquisas empíricas uma vez que existe espaço a ser preenchido por resultados baseados nesse tipo de metodologia.

\subsection{Análise dos artigos publicados no periódico Gestão \& Produção}

Para fins ilustrativos, submeteram-se sob os mesmos critérios os artigos publicados no periódico Gestão \& Produção entre o ano de 2004 e 2006. Não se procedeu à análise relativa à relevância e qualidade

Tabela 2. Classificação dos artigos do periódico Gestão \& Produção em tópicos de pesquisa.

\begin{tabular}{|c|c|c|c|c|c|c|c|c|c|c|c|}
\hline \multirow[t]{2}{*}{ Área } & \multirow[t]{2}{*}{ Tópico } & \multicolumn{2}{|c|}{2004} & \multirow{2}{*}{$\begin{array}{c}\text { Total } \\
(2004)\end{array}$} & \multicolumn{2}{|c|}{2005} & \multirow{2}{*}{$\begin{array}{c}\text { Total } \\
(2005) \\
\end{array}$} & \multicolumn{2}{|c|}{2006} & \multirow{2}{*}{$\begin{array}{c}\text { Total } \\
(2006) \\
\end{array}$} & \multirow{2}{*}{$\begin{array}{l}\text { Total } \\
\text { geral }\end{array}$} \\
\hline & & $\mathbf{E}$ & M & & $\mathbf{E}$ & M & & $\mathbf{E}$ & $\mathbf{M}$ & & \\
\hline $\begin{array}{l}\text { Antecedentes } \\
\text { e orientação da } \\
\text { empresa }\end{array}$ & Implementação da CGS & 1 & & 1 & & & & & & & 1 \\
\hline \multicolumn{2}{|c|}{ Antecedentes e orientação da empresa (total) } & 1 & & 1 & & & & & & & 1 \\
\hline \multirow{5}{*}{$\begin{array}{l}\text { Atividades } \\
\text { da gestão } \\
\text { da cadeia de } \\
\text { suprimentos } \\
\text { (GCS) }\end{array}$} & Agilidade na GCS & 1 & & 1 & & & & & & & 1 \\
\hline & Coordenação & 1 & 2 & 3 & 1 & & 1 & 1 & & 1 & 5 \\
\hline & Design da cadeia & 3 & & 3 & 1 & & 1 & & 1 & 1 & 5 \\
\hline & Inventário & & & & 1 & 1 & 2 & & & & 2 \\
\hline & Planejamento de produção & 1 & & 1 & & & & & & & 1 \\
\hline \multirow{2}{*}{\multicolumn{2}{|c|}{$\begin{array}{l}\text { Troca de informações } \\
\text { Atividades da gestão da cadeia de } \\
\text { suprimentos (GCS) total }\end{array}$}} & 1 & & 1 & 1 & 1 & 2 & & & & 3 \\
\hline & & 7 & 2 & 9 & 4 & 2 & 6 & 1 & 1 & 2 & 17 \\
\hline \multirow[t]{4}{*}{ Consequências } & Desempenho & 3 & 1 & 4 & & & & & & & 4 \\
\hline & Redução de custos & & 1 & 1 & & & & & & & 1 \\
\hline & nsequências (total) & 3 & 2 & 5 & & & & & & & 5 \\
\hline & Total geral & 11 & 4 & 15 & 4 & 2 & 6 & 1 & 1 & 2 & 23 \\
\hline
\end{tabular}

Fonte: Autores (2010). 
uma vez que tal aspecto consiste em uma avaliação do periódico em si e não dos artigos que o compõem. A Tabela 2 apresenta resumidamente o enquadramento dos artigos analisados.

A Tabela 2 organizou os artigos no modelo proposto (Figura 1) por Mentzer et al. (2001) e nas abordagens de pesquisa propostos por Vasilis et al. (2007). Assim sendo, é possível observar o quantitativo de artigos em cada um dos tópicos de pesquisa conforme as variáveis e agrupamentos propostos por Mentzer et al. (2001). A Tabela 3 procura sintetizar as informações em função dos tópicos.

Como pode ser observado na Tabela 3, 74\% dos artigos analisados concentram-se nas Atividades da Gestão da Cadeia de Suprimentos (GCS). Tal aspecto é semelhante aos resultados encontrados na análise dos artigos de periódicos internacionais. Contudo, deve-se ressalvar o quantitativo de periódicos internacionais analisados comparativamente a uma única amostra dentre periódicos nacionais. Em linhas gerais, pode-se afirmar certa proximidade dos interesses de produção apresentados na Tabela 1 e Tabela 3. Por um lado, chama a atenção o baixo percentual relativo de pesquisas em relação ao tópico de Troca de Informações entre os artigos nacionais e internacionais. Por outro lado, há um maior interesse, no caso brasileiro, sobre questões relativas ao design da cadeia de suprimentos.

Por sua vez a Tabela 4 apresenta a distribuição de trabalhos com abordagem empírica e de modelagem. Nesse aspecto a produção se apresenta eminentemente empírica. Tal característica pode, sob certo ponto de vista, representar o aspecto eminentemente aplicado da área de Engenharia de Produção no Brasil. Contudo, tais conclusões devem ser relativizadas, pois o recorte para seleção dos periódicos analisados não teve como critério principal as áreas de conhecimento para as quais tais periódicos representam a produção científica.

As análises, anteriormente realizadas, parecem apontar para um conjunto de oportunidades de produção de conhecimento em áreas e tópicos ainda pouco explorados. Em termos de abordagem também parece haver espaço significativo para o desenvolvimento de pesquisas que utilizem ferramentas de modelagem para a geração de conhecimento. Além disso, de acordo com a análise realizada, há uma oportunidade efetiva para a publicação da produção

Tabela 3. Síntese das Áreas e Tópicos segundo o modelo Mentzer et al. (2001).

\begin{tabular}{lccc}
\hline \multicolumn{1}{c}{ Área } & Tópico & $\begin{array}{c}\text { Número de } \\
\text { artigos }\end{array}$ & $\begin{array}{c}\text { Frequência } \\
(\boldsymbol{\%})\end{array}$ \\
\hline Antecedentes e orientação da empresa & Implementação da CGS & 1 & 4 \\
Antecedentes e orientação da empresa (total) & Agilidade na GCS & 1 & 4 \\
Atividades da gestão da cadeia de & Coordenação & 1 & 4 \\
suprimentos (GCS) & Design da cadeia & 5 & 22 \\
& Inventário & 5 & 22 \\
& Planejamento de produção & 2 & 9 \\
Atividades da gestão da cadeia de suprimentos (GCS) total & 1 & 4 \\
Consequências & Desempenho & 3 & 13 \\
& Redução de custos & 17 & 74 \\
& & 4 & 17 \\
& Total geral & 1 & 4 \\
\hline
\end{tabular}

Fonte: Autores (2010).

Tabela 4. Síntese das abordagens metodológicas utilizadas.

\begin{tabular}{cccccc}
\hline & & Absoluto & \multicolumn{2}{c}{ Relativo } \\
\hline Ano & Empírico & Modelagem & Total geral & Empírica (\%) & Modelagem $(\%)$ \\
\hline 2004 & 11 & 4 & 15 & 73 & 27 \\
2005 & 4 & 2 & 6 & 67 & 33 \\
2006 & 1 & 1 & 2 & 50 & 50 \\
Total geral & 16 & 7 & 23 & 70 & 30 \\
\hline
\end{tabular}

Fonte: Autores (2010). 
científica nacional nos periódicos internacionais. Para tanto, é necessário observar os periódicos que apresentam maior sinergia, em termos de assuntos e abordagens metodológicas, com as características da pesquisa brasileira. A seguir são tecidas as conclusões do presente artigo.

\section{Conclusões}

Esse trabalho teve o objetivo de analisar os artigos sobre gestão da cadeia de suprimentos publicados nos principais periódicos acadêmicos da área de produção e operações nos anos de 2004, 2005 e 2006. Os principais tópicos que englobam a gestão da cadeia de suprimentos foram analisados em cada artigo utilizado na amostra. Os resultados mostraram quais tópicos foram mais pesquisados e quais ainda são carentes de pesquisa, servindo de oportunidade para futuros estudos. Além disso, se apresentou, brevemente, o que tem sido estudado nos principais tópicos da área. Espera-se que tais descrições possam servir de referência para que futuros pesquisadores e estudantes. Além disso, se espera que despertem seu interesse pelos temas apresentados.

A gestão da cadeia de suprimentos mostrou-se um tema complexo que envolve um conjunto elevado de variáveis e relacionamentos, no qual tais variáveis têm reflexos em diversas empresas simultaneamente. Por essa razão, apesar da quantidade de artigos analisados, ainda há um amplo espaço de pesquisa sobre o tema. Existem relações entre as variáveis ainda não explicadas. Existem variáveis ainda não exploradas. Novos modelos teóricos são necessários para aprimorar o conhecimento até então desenvolvido. Essas proposições ajudam a mostrar que a área de gestão da cadeia de suprimentos ainda é jovem e não se consolidou como disciplina, conforme a introdução desse artigo evidenciou.

Esse trabalho também pode mostrar as características de alguns periódicos acadêmicos internacionais. Utilizando o gráfico apresentado na Figura 2, foi possível posicionar os artigos analisados dentro das dimensões características do periódico e metodologia empregada. Essas dimensões podem auxiliar pesquisadores e estudantes no planejamento de suas pesquisas e na seleção de periódicos acadêmicos. Por exemplo, trabalhos empíricos podem ter maiores chances de publicação em periódicos como Journal of Operations Management, International Journal of Operations and Production Management e Productions and Operations Managment.

Uma das limitações deste trabalho é a composição da amostra que levou em consideração apenas periódicos acadêmicos internacionais e da área de produção e operações. Periódicos acadêmicos de outras áreas tais como marketing e estratégia também podem ter estudos sobre gestão da cadeia de suprimentos que não foram considerados neste trabalho.
Outra limitação diz respeito ao modelo utilizado como referência para classificar e analisar os tópicos de pesquisa em gestão da cadeia de suprimentos. Esse modelo não reflete por completo o fenômeno da cadeia de suprimentos, embora seja difícil encontrar algum modelo que consiga reproduzir por completo um fenômeno real. Outros modelos podem agregar, complementar e discutir o modelo utilizado.

A classificação dos estudos feitos em gestão da cadeia de suprimentos também não consegue refletir a total dimensão dos estudos realizados. Em outras palavras, na tentativa de encaixar os trabalhos realizados dentro do modelo utilizado, algumas simplificações foram realizadas, embora não se perca a integridade e contribuição de cada estudo. Alguns artigos trataram de mais de um tópico, o que não foi levado em consideração neste trabalho.

A quantidade de artigos e de tópicos analisados, juntamente com as limitações de espaço de publicação inerentes a um artigo científico, impediu análises aprofundas dos resultados de cada artigo. Infelizmente, esse é um trade-off inerente ao tipo de trabalho proposto e apresentado neste artigo. Uma quantidade menor de artigos poderia ter sido analisada aprofundadamente, proporcionando maior riqueza de detalhe sobre os resultados alcançados, porém perder-se-ia uma perspectiva mais ampla, que foi o objetivo deste artigo. Futuros trabalhos poderão focar somente tópicos específicos aprofundando as análises iniciadas aqui.

\section{Referências}

AHN, H. S.; KAMINSKY, P. Production and distribution policy in a two-stage stochastic push-pull supply chain. IIE Transactions, v. 37, n. 7, p. 609-621, 2005.

BARRATT, M.; OKE, A. Antecedents of supply chain visibility in retail suply chains: a resource-based theory perspective. Journal of Operations Management, v. 25, n. 6, p. 1217-1233, 2007.

BERNSTEIN, F.; FEDERGRUEN, A. Decentralized supply chains with competing retailers under demand uncertainty. Management Science, v. 51, n. 1, p. 18-29, 2005.

BERNSTEIN, F.; CHEN, F.; FEDERGRUEN, A. Coordinating supply chains with simple pricing schemes: the role of vendor-managed inventories. Management Science, v. 52, n. 10, p. 1483-1492, 2006.

BOYACI, T. Competitive stocking and coordination in a multiple-channel distribution system. IIE Transactions, v. 37, n. 5, p. 407-427, 2005.

BOYACI, T.; GALLEGO, B. Supply chain coordination in a market with customer service competition. Production and Operations Management, v. 13, n. 1, p. 3-22, 2004.

BURGESS, K.; SINGH, P. J.; KOROGLU, R. Supply chain management: a structured literature review and implications for future research. International Journal of Operations; Production Management, v. 26, n. 7, p. 703-729, 2006. 
CAGLIANO, R.; CANIATO, F.; SPINA, G. E-business strategy: how companies are shaping their supply chain through the internet. International Journal of Operations; Production Management, v. 25, n. 12, p. 1309-1327, 2005.

CATTANI, K.; GILLAND, W.; HEESE, H. S. et al. Boiling frogs: pricing strategies for a manufacturer adding a direct channel that competes with the traditional channel. Production and Operations Management, v. 15, n. 1, p. 40-56, 2006.

CHEN, I. J.; PAULRAJ, A. Towards a theory of supply chain management: the constructs and measurements. Journal of Operations Management, v. 22, n. 2, p. 119-150, 2004.

CHU, W. H. J.; LEE, C. C. Strategic information sharing in a supply chain. European Journal of Operational Research, v. 174, n. 3, p. 1567-1579, 2006.

CHOI, T. Y.; KRAUSE, D. R. The supply base and its complexity: implications for transaction costs, risks, responsiveness, and innovation. Journal of Operations Management, v. 24, n. 5, p. 637-652, 2006.

COUSINS, P. D.; HANDFIELD, R. B.; LAWSON, B. et al. Creating supply chain relational capital: The impact of formal and informal socialization processes. Journal of Operations Management, v. 24, n. 6, p. 851-863, 2006.

CROOM, S. R. The impact of e-business on supply chain management: an empirical study of key developments. International Journal of Operations; Production Management, v. 25, n. 1, p. 55-73, 2005.

DANIEL, J. S. R.; RAJENDRAN, C. Heuristic approaches to determine base-stock levels in a serial supply chain with a single objective and with multiple objectives. European Journal of Operational Research, v. 175, n. 1, p. 566-592, 2006.

DAWANDE, M.; GEISMAR, H. N.; HALL, N. G. et al. Supply chain scheduling: distribution systems. Production and Operations Management, v. 15, n. 2, p. 243-261, 2006.

DUDEK, G.; STADTLER, H. Negotiation-based collaborative planning between supply chains partners. European Journal of Operational Research, v. 163, n. 3, p. 668-687, 2005.

FIXSON, S. F. Product architecture assessment: a tool to link product, process, and supply chain design decisions. Journal of Operations Management, v. 23, n. 3-4, p. 345-369, 2005.

GATTIKER, T. F.; HUANG, X.; SCHWARZ, J. L. Negotiation, email, and Internet reverse auctions: how sourcing mechanisms deployed by buyers affect suppliers' trust. Journal of Operations Management, v. 25, n. 1, p. 184-202, 2007.

GRAHAM, G.; BURNES, B.; LEWIS, G. J. et al. The transformation of the music industry supply chain: a major label perspective. International Journal of Operations; Production Management, v. 24, n. 11, p. 1087-1103, 2004.

GÜLLÜ, R.; HOUTUM, G. J. V.; SARGUT, F. Z. et al. Analysis of a decentralized supply chain under partial cooperation. Manufacturing; Service Operations Management, v. 7, n. 3, p. 229-247, 2005.
HARLAND, C. M.; LAMMING, R. C.; COUSINS, P. D. Developing the concept of supply strategy. International Journal of Operations; Production Management, v. 19, n. 7, p. 650-669, 1999.

HARLAND, C. M.; LAMMING, R. C.; WALKER, H. et al. Supply management: is it a discipline? International Journal of Operations; Production Management, v. 26, n. 7, p. 730-753, 2006.

HARLAND, C. M.; CALDWELL, N. D.; POWELL, P. et al. Barriers to supply chain information integration: SMEs adrift of eLands. Journal of Operations Management, v. 25, n. 6, p. 1234-1254, 2007.

HAZRA, J.; MAHADEVAN, B.; SESHADRI, S. Capacity allocation among multiple suppliers in an eletronic market. Production and Operations Management, v. 13, n. 2, p. 161-170, 2004.

HENDRICKS, K. B.; SINGHAL, V. R. Association between supply chain glitches and operating performance. Management Science, v. 51, n. 5, p. 695-711, 2005a.

HENDRICKS, K. B.; SINGHAL, V. R. An empirical analysis of the effect of supply chain disruptions on long-run stock price performance and equity risk of the firm. Production and Operations Management, v. 14, n. 1, p. 35-52, 2005 b.

HULT, G. T. M.; KETCHEN Jr., D. J.; CAVUSGIL, S. T. et al. Knowledge as a strategic resource in supply chains. Journal of Operations Management, v. 24, n. 1, p. 458-475, 2006.

IRELAND, R. D.; WEBB, J. W. A multi-theoretic perspective on trust and power in strategic supply chains. Journal of Operations Management, v. 25, n. 2, p. 482-497, 2007.

JOHNSTON, D. A.; MCCUTCHEON, D. M.; STUART, F. I. et al. Effect of supplier trust on performance of cooperative supplier relationships. Journal of Operations Management, v. 22, n. 1, p. 23-38, 2004.

LAMOTHE, J.; HADJ-HAMOU, K.; ALDANONDO, M. An optimization model for selecting a product family and designing its supply chain. European Journal of Operational Research, v. 169, n. 3, p. 1030-1047, 2006.

LI, S.; RAO, S. S.; RAGU-NATHAN, T. S. et al. Development and validation of a measurement instrument for studying supply chain management practices. Journal of Operations Management, v. 23, n. 6, p. 618-641, 2005.

LINTON, J. D.; KLASSEN, R.; JAYARAMAN, V. Sustainable supply chains: an introduction. Journal of Operations Management, v. 25, n. 6, p. 1075-1082, 2007.

LOCH, C. H.; TERWIESCH, C. Rush and be wrong or wait and be late? A model of information in collaborative processes. Production and Operations Management, v. 14, n. 3, p. 331-343, 2005.

MARQUEZ, A. C.; BIANCHI, C.; GUPTA, J. N. D. Operational and financial effectiveness of e-collaboration tools in supply chain integration. European Journal of Operational Research, v. 159, n. 2, p. 348-363, 2004. 
MCCARTER, M. W.; NORTHCRAFT, G. B. Happy together? Insights and implications of viewing managed supply chains a social dilemma. Journal of Operations Management, v. 25, n. 2, p. 498-511, 2007.

MENTZER, J.; DEWITT, W.; KEEBLER, J. S. et al. Defining supply chain management. Journal of Business Logistics, v. 22, n. 2, p. 1-25, 2001.

MIYAOKA, J.; HAUSMAN, W. How a base stock policy using "stale" forecasts provides supply chain benefits. Manufacturing; Service Operations Management, v. 6, n. 2, p. 149-162, 2004.

NAGURNEY, A.; CRUZ, J.; DONG, J. et al. Supply chain networks, electronic commerce, and supply side and demand risk. European Journal of Operational Research, v. 164, n. 1, p. 120-142, 2005.

PERSONA, A.; REGATTIERI, A.; PHAM, H. et al. Remote control and maintenance outsourcing networks and its applications in supply chain management. Journal of Operations Management, v. 25, n. 6, p. 1275-1291, 2007.

PIDD, M. Modelagem empresarial: ferramentas para tomada de decisão. Porto Alegre: Bookman, 1998.

POULIN, M.; MONTREUIL, B.; MARTEL, A. Implications of personalization offers on demand and supply network design: A case from the golf club industry. European Journal of Operational Research, v. 169, n. 3, p. 996-1009, 2006.

POUNDARIKAPURAM, S.; VEERAMANI, D. Distributed decision-making in supply chains and private e-marketplaces. Production and Operations Management, v. 13, n. 1, p. 111-121, 2004.

RAY, S.; LI, S.; SONG, Y. Tailored supply chain decision making under price-sensitive stochastic demand and delivery uncertainty. Management Science, v. 51, n. 12, p. 1873-1891, 2005

RAY, S.; GERCHAK, Y.; JEWKES, E. M. The effectiveness of investment in lead time production for a make-tostock product. IIE Transactions, v. 36, n. 4, p. 333-344, 2004.

RAO, U. S.; SWAMINATHAN, J. M.; ZHANG, J. Demand and production management with uniform guaranteed lead time. Production and Operations Management, v. 14, n. 4, p. 400-412, 2005.

SAEED, K. A.; MALHOTRA, M. K.; GROVER, V. Examining the impact of interorganizational systems on process efficiency and sourcing leverage in buyer supplier dyads. Decision Sciences, v. 36, n. 3, p. 365-396, 2005.

SANDERS, N. R. An empirical study of the impact of e-business technologies on organizational collaboration and performance. Journal of Operations Management, v. 25 , n. 6, p. 1332-1347, 2007.

SEIFERT, R. W.; THONEMANN, U. W.; SEIKE, M. A. Relaxing channel separation: integrating a virtual store into the supply chain via transshipments. IIE Transactions, v. 38, n. 11, p. 917-931, 2006.

SELVARAJAH, E.; STEINER, G. Batch scheduling in a two-level supply chain - a focus on the supplier. European Journal of Operational Research, v. 173, n. 1, p. 226-240, 2006.

SHEU, J. B. A multi-layer demand-responsive logistics control methodology for alleviating the bullwhip effect of supply chains. European Journal of Operational Research, v. 161, n. 3, p. 797-811, 2005.
SIMCHI-LEVI, D.; ZHAO, Y. Safety stock positioning in supply chains with stochastic lead times. Manufacturing; Service Operations Management, v. 7, n. 4, p. 295-318, 2005.

SIVADASAN, S.; EFSTATHIOU, J.; CALINESCU, A. et al. Advances on measuring the operational complexity of supplier-customer systems. European Journal of Operational Research, v. 171, n. 1, p. 208-226, 2006.

SOBEL, M.; FILL, J. Rates of single-stage and multistage supply systems. Manufacturing; Service Operations Management, v. 6, n. 1, p. 41-52, 2004.

SOUZA, G. C.; ZHAO, Z.; CHEN, M. et al. Coordinating sales and raw material discounts in a global supply chain. Production and Operations Management, v. 13, n. 1, p. 34-45, 2004.

STADTLER, H. Supply chain management and advanced planning-basics, overview and challenges. European Journal of Operational Research, v. 163, n. 3, p. 575-588, 2005.

STOREY, J.; EMBERSON, C.; GODSELL, J. et al. Supply chain management: theory, practice and future challenges. International Journal of Operations; Production Management, v. 26, n. 7, p. 754-774, 2006.

SWAFFORD, P. M.; GHOSH, S.; MURTHY, N. The antecedents of supply chain agility of a firm: scale development and model testing. Journal of Operations Management, v. 24, n. 2, p. 170-188, 2006.

TAN, K. C. A framework f supply chain management literature. European Journal of Purchasing and Supply Management, v. 7, n. 1, p. 39-48, 2001.

THEMISTOCLEOUS, M.; IRANI, Z.; LOVE, P. E. D Evaluating the integration of suppl y chain information systems: A case study. European Journal of Operational Research, v. 159, n. 2, p. 393-405, 2004.

VAART, T.; DONK, D. P. A critical review of survey-based research in supply chain integration. International Journal of Production Economics, v. 111, n. 1, p. 42-55, 2008.

VASILIS, T.; VOSS, C.; HADJINICOLA, G. C. et al. Insight factors affecting production and operations management (POM) journal evaluation. Journal of Operations Management, v. 25, n. 4, p. 932-948, 2007.

YUE, J.; AUSTIN, J.; WANG, M. C. et al. Coordination of cooperative advertising in a two-level supply chain when manufacturer offers discounts. European Journal of Operational Research, v. 168, n. 1, p. 65-85, 2006.

WANG, S.; SARKER, B. R. Optimal models for a multi-stage supply chain system controlled by kanban under justin-time philosophy. European Journal of Operational Research, v. 172, n. 1, p. 179-200, 2006.

WANG, H.; GUO, M.; EFSTATHIOU, J. A game-theoretical cooperative mechanism design for a two-echelon decentralized supply chain. European Journal of Operational Research, v. 157, n. 2, p. 372-388, 2004.

WU, Z.; CHOI, T. Y. Supplier-supplier relationships in the buyer-supplier triad: building theories from eight case studies. Journal of Operations Management, v. 24, n. 1, p. 27-52, 2005.

ZHOU, H.; BENTON Jr., W. C. Supply Chain Practice and Information Sharing. Journal of Operations Management, v. 25, n. 6, p. 1348-1365, 2007. 


\section{Anexo I - Artigos Internacionais Analisados}

AHN, H. S.; KAMINSKY, P. Production and distribution policy in a two-stage stochastic push-pull supply chain. IIE Transactions, v. 37, p. 609-621, 2005.

ANDERSON, E. G.; MORRICE, D. J.; WANG, I. J. Stochastic optimal control for staffing and backlog policies in a two-stage customized service supply chain. Production and Operations Management, v. 15, n. 2, p. 262-278, 2006.

ARCELUS, F. J.; KUMAR, S.; SRINIVASAN, G. Retailer's response to alternate manufacturer's incentives under a single-period, price-dependent, stochastic-demand framework. Decision Sciences, v. 36, n. 4, p. 599-626, 2005.

BAINES, T. et al. Strategic positioning: an integrated decision process for manufacturers. International Journal of Operations \& Production Management, v. 24, n. 10, p. 1037-1054, 2004.

BALAKRISHNAN, A.; GEUNES, J. Collaboration and coordination in supply chain management and e-commerce. Production and Operations Management, v. 13, n. 1, p. 1-2, 2004.

BARRATT, M.; OKE, A. Antecedents of supply chain visibility in retail suply chains: a resource-based theory perspective. Journal of Operations Management, v. 25, p. 1217-1233, 2007.

BEN-TAL, A. Retailer-supplier flexible commitments contracts: a robust optimization approach. Manufacturing \& Service Operations Management, v. 7, n. 3, p. 248-271, 2005.

BERLING, P.; MARKLUND, J. Heuristic coordination of decentralized inventory systems using induced backorder costs. Production and Operations Management, v. 15, n. 2, p. 294-310, 2006.

BERNSTEIN, F.; CHEN, F.; FEDERGRUEN, A. Coordinating supply chains with simple pricing schemes: the role of vendor-managed inventories. Management Science, v. 52, n. 10, p. 1483-1492, 2006.

BERNSTEIN, F.; DECROIX, G. A. Inventory policies in a decentralized assembly system. Opertions Research, v. 54, n. 2, p. 324-336, 2006.

BERNSTEIN, F.; FEDERGRUEN, A. Decentralized supply chains with competing retailers under demand uncertainty. Management Science, v. 51, n. 1, p. 18-29, 2005.

BISH, E. K.; MURIEL, A.; BILLER, S. Managing flexible capacity in a make-to-order environment. Management Science, v. 51, n. 2, p. 167-180, 2005.

BLACKHURST, J.; WU, T.; O'GRADY, P. PCDM: a decision support modeling methodology for supply chain, product and process design decisions. Journal of Operations Management, v. 23, p. 325-343, 2005.

BOLLAPRAGADA, R.; RAO, U. S.; ZHANG, J. Managing two-stage serial inventory systems under demand and supply uncertainty and customer service level requirements. IIE Transactions, v. 36, n. 4, p. 73-85, 2004.

BOYACI, T. Competitive stocking and coordination in a multiple-channel distribution system. IIE Transactions, v. 37 , p. 407-427, 2005.

BOYACI, T.; GALLEGO, B. Supply chain coordination in a market with customer service competition. Production and Operations Management, v. 13, n. 1, p. 3-22, 2004.

BREDSTRÖM, D. et al. Supply chain optimization in the pulp mill industry - IP models, column generation and novel constraint branches. European Journal of Operational Research, v. 156, p. 2-22, 2004.

BRUCE, M.; TOWERS, L. D. N. Lean or agile: a solution for supply chain management in the textiles and clothing industry? International Journal of Operations \& Production Management, v. 24, n. 2, p. 151-170, 2004.

CACHON, G. P. The allocation of inventory risk in a supply chain: push, pull, and advance-purchase discount contracts. Management Science, v. 50, n. 2, p. 222-238, 2004.

CACHON, G. P.; LARIVIERE, M. A. Supply chain coordination with revenue-sharing contracts: strengths and limitations. Management Science, v. 51, n. 1, p. 30-44, 2005.

CAGGIANO, K. E.; MUCKSTADT, J. A.; RAPPOLD, J. A. Integrated real-time capacity and inventory allocation for reparable service parts in a two-echelon supply system. Manufacturing \& Service Operations Management, v. 8, n. 3, p. 292-319, 2006.

CAGLIANO, R.; CANIATO, F.; SPINA, G. E-business strategy: how companies are shaping their supply chain through the internet. International Journal of Operations \& Production Management, v. 25, n. 12, p. 1309-1327, 2005.

CAGLIANO, R.; CANIATO, F.; SPINA, G. The linkage between supply chain integration and manufacturing improvement programmes. International Journal of Operations \& Production Management, v. 26, n. 3, p. 282-299, 2006.

CARLSSON, D.; RÖNNQVIST, M. Supply chain management in forestry - case studies at Södra Cell AB. European Journal of Operational Research, v. 163, p. 589-616, 2005.

CATTANI, K. et al. Boiling frogs: pricing strategies for a manufacturer adding a direct channel that competes with the traditional channel. Production and Operations Management, v. 15, n. 1, p. 40-56, 2006.

CHATFIELD, D. C. et al. The Bullwhip Effect: impact of stochastic lead time, information quality, and information sharing: a simulation study. Production and Operations Management, v. 13, n. 4, p. 340-353, 2004.

CHEN, F.; SAMROENGRAJA, R. Order volatility and supply chain costs. Operations Research, v. 52, n. 5, p. 707-722, 2004.

CHEN, H.; FRANK, M. Z.; WU, O. Q. What actually happened to the inventories of American companies between 1981 and 200? Management Science, v. 51, n. 7, p. 1015-1031, 2005. 
CHEN, Z. L.; PUNDOOR, G. Order assignment and scheduling in a supply chain. Operations Research, v. 54, n. 3 , p. 555-572, 2006.

CHOI, T. Y.; KRAUSE, D. R. The supply base and its complexity: implications for transaction costs, risks, responsiveness, and innovation. Journal of Operations Management, v. 24, p. 637-652, 2006.

CHOPRA, S.; REINHARDT, G.; DADA, M. The effect of lead time uncertainty on safety stocks. Decision Sciences, v. 35, n. 1, p. 1-24, 2004.

CHRISTENSEN, W. J.; GERMAIN, R.; BIROU, L. Build-to-order and just-in-time as predictors of applied supply chain knowledge and market performance. Journal of Operations Management, v. 23, p. 470-481, 2005.

CHU, W. H. J.; LEE, C. C. Strategic information sharing in a supply chain. European Journal of Operational Research, v. 174, p. 1567-1579, 2006.

CIGOLINI, R.; COZZI, M.; PERONA, M. A new framework for supply chain management: conceptual model and empirical test. International Journal of Operations \& Production Management, v. 24, n. 1, p. 7-41, 2004.

CORBETT, C. J.; DECROIX, G. A.; HA, A. Y. Optimal shared-savings contracts in supply chains: linear contracts and double moral hazard. European Journal of Operational Research, v. 163, n. 653-667, 2005.

COUSINS, P. D. The alignment of appropriate firm and supply strategies for competitive advantage. International Journal of Operations \& Production Management, v. 25, n. 5, p. 403-428, 2005.

COUSINS, P. D. et al. Creating supply chain relational capital: The impact of formal and informal socialization processes. Journal of Operations Management, v. 24, p. 851-863, 2006.

COUSINS, P. D.; LAMMING, R. C.; BOWEN, F. The role of risk in environmental-related supplier initiatives. International Journal of Operations \& Production Management, v. 24, n. 4, p. 554-565, 2004.

COUSINS, P. D.; MENGUC, B. The implications of socialization and integration in supply chain management. Journal of Operations Management, v. 24, p. 604-620, 2006.

CROOM, S. R. The impact of e-business on supply chain management: an empirical study of key developments. International Journal of Operations \& Production Management, v. 25, n. 1, p. 55-73, 2005.

DANIEL, J. S. R.; RAJENDRAN, C. Heuristic approaches to determine base-stock levels in a serial supply chain with a single objective and with multiple objectives. European Journal of Operational Research, v. 175, p. 566-592, 2006.

DAS, A.; NARASIMHAN, R.; TALLURI, S. Supplier integration - finding an optimal configuration. Journal of Operations Management, v. 24, p. 563-582, 2006.

DAWANDE, M. et al. Supply chain scheduling: distribution systems. Production and Operations Management, v. 15, n. 2, p. 243-261, 2006.

DECROIX, G. A. Optimal policy for multiechelon inventory system with remanufacturing. Operations Research, v. 54, n. 3, p. 532-543, 2006.

DEJONCKHEERE, J. et al. The impact of information enrichment on the bullwhip effect in supply chains: a control engineering perspective. European Journal of Operational Research, v. 153, p. 727-750, 2004.

DUDEK, G.; STADTLER, H. Negotiation-based collaborative planning between supply chains partners. European Journal of Operational Research, v. 163, p. 668-687, 2005.

FERRER, G.; KETZENBERG, M. E. Value of information in remanufacturing complex products. IIE Transactions, v. 36, p. 265-277, 2004.

FIXSON, S. F. Product architecture assessment: a tool to link product, process, and supply chain design decisions. Journal of Operations Management, v. 23, p. 345-369, 2005.

FRENCH, M. L.; LAFORGE, R. L. Closed-loop supply chains in process industries: an empirical study of producer re-use issues. Journal of Operations Management, v. 24, n. 271-286, 2006.

FU, Y.; PIPLANI, R. Supply-side collaboration and its value in supply chains. European Journal of Operational Research, v. 152, p. 281-288, 2004.

FYNES, B.; VOSS, C.; BÚRCA, S. The impact of supply chain relationship dynamics on manufacturing performance. International Journal of Operations \& Production Management, v. 25, n. 1, p. 6-19, 2005.

GAN, X.; SETHI, S. P.; YAN, H. Coordination of supply chains with risk-averse agents. Production and Operations Management, v. 13, n. 2, p. 135-149, 2004.

GATTIKER, T. F.; HUANG, X.; SCHWARZ, J. L. Negotiation, email, and Internet reverse auctions: how sourcing mechanisms deployed by buyers affect suppliers' trust. Journal of Operations Management, v. 25, p. 184-2002, 2007.

GAVIRNENI, S. Price fluctuations, information sharing, and supply chain performance. European Journal of Operational Research, v. 174, p. 1651-1663, 2006.

GERCHAK, Y.; WANG, Y. Revenue-sharing vs. wholesale-price contracts in assembly systems with random demand. Production and Operations Management, v. 13, n. 1, p. 23-33, 2004.

GIUNIPERO, L.; HANDFIELD, R. B.; ELTANTAWY, R. Supply management's evolution: key skill sets for the supply manager of the future. International Journal of Operations \& Production Management, v. 26, n. 7, p. 822-844, 2006.

GRAHAM, G. et al. The transformation of the music industry supply chain: a major label perspective. International Journal of Operations \& Production Management, v. 24, n. 11, p. 1087-1103, 2004. 
GRAVES, S. C.; WILLEMS, S. P. Optimizing the supply chain configuration for new products. Management Science, v. 51, n. 8, p. 1165-1180, 2005.

GÜLLÜ, R. et al. Analysis of a decentralized supply chain under partial cooperation. Manufacturing \& Service Operations Management, v. 7, n. 3, p. 229-247, 2005.

GUNASEKARAN, A.; NGAI, E. W. T. Information system in supply chain integration and management. European Journal of Operational Research, v. 159, p. 269-295, 2004.

GUNNARSSON, H.; RÖNNQVIST, M.; LUNDGREN, J. T. Supply chain modeling of forest fuel. European Journal of Operational Research, v. 158, p. 103-123, 2004.

GUPTA, D.; SELVARAJU, N. Performance evaluation and stock allocation in capacitated serial supply systems. Manufacturing \& Service Operations Management, v. 8, n. 2, p. 169-191, 2006.

HARLAND, C. M. et al. Barriers to supply chain information integration: SMEs adrift of eLands. Journal of Operations Management, v. 25, p. 1234-1254, 2007.

HAZRA, J.; MAHADEVAN, B.; SESHADRI, S. Capacity allocation among multiple suppliers in an eletronic market. Production and Operations Management, v. 13, n. 2, p. 161-170, 2004.

HEESE, H. S. et al. Competitive advantage through take-back of used products. European Journal of Operational Research, v. 164, p. 143-157, 2005.

HENDRICKS, K. B.; SINGHAL, V. R. Association between supply chain glitches and operating performance. Management Science, v. 51, n. 5, p. 695-711, 2005a.

HENDRICKS, K. B.; SINGHAL, V. R. An empirical analysis of the effect of supply chain disruptions on long-run stock price performance and equity risk of the firm. Production and Operations Management, v. 14, n. 1, p. 35-52, 2005b.

HOLWEG, M. The three dimensions of responsiveness. International Journal of Operations \& Production Management, v. 25, n. 7, p. 603-622, 2005.

HOPP, W. J.; SPEARMAN, M. L. To pull or not to pull: what is the question? Manufacturing \& Service Operations Management, v. 6, n. 2, p. 133-148, 2004

HUANG, B.; IRAVANI, S. M. R. Production control policies in supply chains with selective-information sharing. Operations Research, v. 53, n. 4, p. 662-674, 2005.

HULT, G. T. M. et al. Knowledge as a strategic resource in supply chains. Journal of Operations Management, v. 24, p. 458-475, 2006.

HUNG, W. Y.; SAMSATLI, N. J.; SHAH, R. Objected-oriented dynamic supply chain modeling incorporated with production scheduling. European Journal of Operational Research, v. 169, p. 1064-1076, 2006.

IRELAND, R. D.; WEBB, J. W. A multi-theoretic perspective on trust and power in strategic supply chains. Journal of Operations Management, v. 25, n. 2, p. 482-497, 2007.

JAYARAMAN, V.; KLASSEN, R.; LINTON, J. D. Supply chain in a sustainable environment. Journal of Operations Management, v. 25, n. 6, p. 1071-1074, 2007.

JOHNSTON, D. A. et al. Effect of supplier trust on performance of cooperative supplier relationships. Journal of Operations Management, v. 22, p. 23-38, 2004.

KARMARKAR, U. S.; APTE, U. M. Operations management in the information economy: information products, processes, and chains. Journal of Operations Management, v. 25, n. 2, p. 438-453, 2007.

KAUFMANN, L.; CARTER, C. R. International supply relationships and non-financial performance - a comparison of U.S. nad German practices. Journal of Operations Management, v. 24, p. 653-675, 2006.

KETCHEN, D. J. J.; HULT, G. T. M. Bridging organization theory and supply chain management: the case of the best value supply chains. Journal of Operations Management, v. 25, n. 2, p. 573-580, 2007a.

KETCHEN, D. J. J.; HULT, G. T. M. Toward greater integration of insights from organization theory and supply chain management. Journal of Operations Management, v. 25, n. 2, p. 455-458, $2007 \mathrm{~b}$.

KIM, S. W. The effect of supply chain integration on the alignment between corporate competitive capability and supply chain operational capability. International Journal of Operations \& Production Management, v. 26, n. 10, p. 1084-1107, 2006.

KLEINDORFER, P. R.; SAAD, G. H. Managing disruptions risks in supply chains. Production and Operations Management, v. 14, n. 1, p. 53-68, 2005.

KLEINDORFER, P. R.; SINGHAL, K.; VAN WASSENHOVE, L. N. Sustainable operations management. Production and Operations Management, v. 14, n. 4, p. 482-492, 2005.

KRAISELBURD, S.; NARAYANAN, V. G.; RAMAN, A. Contracting in a supply chain with stochastic demand and substitute products. Production and Operations Management, v. 13, n. 1, p. 46-62, 2004.

LAMOTHE, J.; HADJ-HAMOU, K.; ALDANONDO, M. An optimization model for selecting a product family and designing its supply chain. European Journal of Operational Research, v. 169, p. 1030-1047, 2006.

LEE, C. C.; CHU, W. H. J. Who should control inventory in a supply chain? European Journal of Operational Research, v. 164, p. 158-172, 2005.

LI, S. et al. Development and validation of a measurement instrument for studying supply chain management practices. Journal of Operations Management, v. 23, p. 618-641, 2005. 
LINTON, J. D.; KLASSEN, R.; JAYARAMAN, V. Sustainable supply chains: an introduction. Journal of Operations Management, v. 25, n. 6, p. 1075-1082, 2007.

LOCH, C. H.; TERWIESCH, C. Rush and be wrong or wait and be late? A model of information in collaborative processes. Production and Operations Management, v. 14, n. 3, p. 331-343, 2005.

LOCKAMY III, A.; MCCORMACK, K. Linking SCOR planning practices to supply chain performance: an exploratory study. International Journal of Operations \& Production Management, v. 24, n. 12, p. 1192-1218, 2004.

LUTZE, H. S.; ÖZER, Ö. Promised leadtime contracts and renegotiation incentives under asymmetric information. Manufacturing \& Service Operations Management, v. 7, n. 1, p. 97-98, 2005.

MAJUMDER, P.; SRINIVASAN, A. Leader location, cooperation, and coordination in serial supply chains. Production and Operations Management, v. 15, n. 1, p. 22-39, 2006.

MALLIK, S.; HARKER, P. T. Coordinating supply chain with competition: capacity allocation in semiconductor manufacturing. European Journal of Operational Research, v. 159, p. 330-347, 2004.

MARQUEZ, A. C.; BIANCHI, C.; GUPTA, J. N. D. Operational and financial effectiveness of e-collaboration tools in supply chain integration. European Journal of Operational Research, v. 159, p. 348-363, 2004.

MCCARTER, M. W.; NORTHCRAFT, G. B. Happy together? Insights and implications of viewing managed supply chains a social dilemma. Journal of Operations Management, v. 25, n. 2, p. 498-511, 2007.

MILES, R. E.; SNOW, C. C. Organization theory and supply chain management: an evolving research perspective. Journal of Operations Management, v. 25, n. 2, p. 459-463, 2007.

MILLS, J.; SCHIMITZ, J.; FRIZELLE, G. A strategic review of "supply networks". International Journal of Operations \& Production Management, v. 24, n. 10, p. 1012-1036, 2004.

MISHRA, B. K.; RAGHUNATHAM, S. Retailer- vs. vendor-managed inventory and brand competition. Management Science, v. 50, n. 4, p. 445-457, 2004.

MIYAOKA, J.; HAUSMAN, W. How a base stock policy using "stale" forecasts provides supply chain benefits. Manufacturing \& Service Operations Management, v. 6, n. 2, p. 149-162, 2004.

NAGURNEY, A. et al. Supply chain networks, electronic commerce, and supply side and demand risk. European Journal of Operational Research, v. 164, p. 120-142, 2005.

NETESSINE, S.; ZHANG, F. Positive vs. negative externalities in inventory management: implications for supply chain design. Manufacturing \& Service Operations Management, v. 7, n. 1, p. 58-73, 2005.

PARK, K. S.; KIM, K. J. Optimizing multi-response surface problems: how to use multi-objective optimization techniques. IIE Transactions, v. 37, p. 523-532, 2005.

PERSONA, A. et al. Remote control and maintenance outsourcing networks and its applications in supply chain management. Journal of Operations Management, v. 25, n. 6, p. 1275-1291, 2007.

POULIN, M.; MONTREUIL, B.; MARTEL, A. Implications of personalization offers on demand and supply network design: A case from the golf club industry. European Journal of Operational Research, v. 169, p. 996-1009, 2006.

POUNDARIKAPURAM, S.; VEERAMANI, D. Distributed decision-making in supply chains and private e-marketplaces. Production and Operations Management, v. 13, n. 1, p. 111-121, 2004.

POWER, D.; SIMON, A. Adoption and difussion in technology implementation: a supply chain study. International Journal of Operations \& Production Management, v. 24, n. 6, p. 566-587, 2004.

RAO, P.; HOLT, D. Do green supply chains lead to competitiveness and economic performance? International Journal of Operations \& Production Management, v. 25, n. 9, p. 898-916, 2005.

RAO, U. S.; SWAMINATHAN, J. M.; ZHANG, J. Demand and production management with uniform guaranteed lead time. Production and Operations Management, v. 14, n. 4, p. 400-412, 2005.

RAY, S.; GERCHAK, Y.; JEWKES, E. M. The effectiveness of investment in lead time production for a make-to-stock product. IIE Transactions, v. 36, p. 333-344, 2004.

RAY, S.; LI, S.; SONG, Y. Tailored supply chain decision making under price-sensitive stochastic demand and delivery uncertainty. Management Science, v. 51, n. 12, p. 1873-1891, 2005.

ROBINSON, E. P. J.; SAHIN, F.; GAO, L. The impact of e-replenishment strategy on make-to-order supply chain performance. Decision Sciences, v. 36, n. 1, p. 33-64, 2005.

SAEED, K. A.; MALHOTRA, M. K.; GROVER, V. Examining the impact of interorganizational systems on process efficiency and sourcing leverage in buyer-supplier dyads. Decision Sciences, v. 36, n. 3, p. 365-396, 2005.

SÁNCHEZ, A. M.; PÉREZ, M. P. Supply chain flexibility and firm performance: a conceptual model and empirical study in the automotive industry. International Journal of Operations \& Production Management, v. 25, n. 7, p. 681-700, 2005.

SANDERS, N. R. An empirical study of the impact of e-business technologies on organizational collaboration and performance. Journal of Operations Management, v. 25, p. 1332-1347, 2007.

SARKIS, J.; TALLURI, S. Evaluating and selecting e-commerce software and communication system for a supply chain. European Journal of Operational Research, v. 159, p. 318-329, 2004.

SCHNEEWEISS, C.; ZIMMER, K. Hierarchical coordination mechanisms within the supply chain. European Journal of Operational Research, v. 153, p. 687-703, 2004. 
SEIFERT, R. W.; THONEMANN, U. W.; SEIKE, M. A. Relaxing channel separation: integrating a virtual store into the supply chain via transshipments. IIE Transactions, v. 38, p. 917-931, 2006.

SELVARAJAH, E.; STEINER, G. Batch scheduling in a two-level supply chain - a focus on the supplier. European Journal of Operational Research, v. 173, p. 226-240, 2006.

SHAH, R.; GOLDSTEIN, S. M. Use of structural equation modeling in operations management research: looking back and forward. Journal of Operations Management, v. 23, p. 618-641, 2005.

SHEU, C.; YEN, H. R.; CHAE, B. Determinants of supplier-retailer collaboration: evidence from an international study. International Journal of Operations \& Production Management, v. 26, n. 1, p. 24-49, 2006.

SHEU, J. B. A multi-layer demand-responsive logistics control methodology for alleviating the bullwhip effect of supply chains. European Journal of Operational Research, v. 161, p. 797-811, 2005.

SHIN, H.; BENTON, W. C. J. Quantity discount-based inventory coordination: effectiveness and critical environmental factors. Production and Operations Management, v. 13, n. 1, p. 63-76, 2004

SIMCHI-LEVI, D.; ZHAO, Y. Safety stock positioning in supply chains with stochastic lead times. Manufacturing \& Service Operations Management, v. 7, n. 4, p. 295-318, 2005.

SIVADASAN, S. et al. Advances on measuring the operational complexity of supplier-customer systems. European Journal of Operational Research, v. 171, p. 208-226, 2006.

SOBEL, M.; FILL, J. Rates of single-stage and multistage supply systems. Manufacturing \& Service Operations Management, v. 6, n. 1, p. 41-52, 2004.

SODHI, M. M. S. Managing demand risk in tactical supply chain planning for a global consumer electronics company. Production and Operations Management, v. 14, n. 1, p. 69-79, 2005.

SOUZA, G. C. et al. Coordinating sales and raw material discounts in a global supply chain. Production and Operations Management, v. 13, n. 1, p. 34-45, 2004.

SPITTER, J. M. et al. Linear programming models with planned lead times for supply chain operations planning. European Journal of Operational Research, v. 163, p. 706-720, 2005.

STADTLER, H. Supply chain management and advanced planning-basics, overview and challenges. European Journal of Operational Research, v. 163, p. 575-588, 2005.

STECKEL, J. H.; GUPTA, S.; BANERJI, A. Supply chain decision making: will shorter cycle times and shared pointof-sale information necessarily help? Management Science, v. 50, n. 4, p. 458-464, 2004.

STONEBRAKER, P. W.; LIAO, J. Environmental turbulence, strategic orientation: modeling supply chain integration. International Journal of Operations \& Production Management, v. 24, n. 10, p. 1037-1054, 2004.

STOREY, J.; EMBERSON, C.; READE, D. The barriers to customer responsive supply chain management. International Journal of Operations \& Production Management, v. 25, n. 3, p. 242-260, 2005.

SZWEJCZEWSKI, M.; LEMKE, F.; GOFFIN, K. Manufacturer-supplier relationships: an empirical study of German manufacturing companies. International Journal of Operations \& Production Management, v. 25, n. 9, p. 875-897, 2005.

THEMISTOCLEOUS, M.; IRANI, Z.; LOVE, P. E. D. Evaluating the integration of suppl y chain information systems: a case study. European Journal of Operational Research, v. 159, p. 393-405, 2004.

THIRUMALAI, S.; SINHA, K. K. Customer satisfaction with order fulfillment in retail supply chains: implications of product type in electronic B2C transactions. Journal of Operations Management, v. 23, p. 291-303, 2005.

TOMLIN, B. On the value of mitigation and contingency strategies for managing supply chain disruption risks. Management Science, v. 52, n. 5, p. 639-657, 2006.

TSAY, A. A.; AGRAWAL, N. Channel conflict and coordination in the e-commerce age. Production and Operations Management, v. 13, n. 1, p. 93-110, 2004.

VAART, T.; DONK, D. P. A critical review of survey-based research in supply chain integration. International Journal of Production Economics, v. 111, p. 42-55, 2008.

VACHON, S.; KLASSEN, R. Extending green practices across the supply chain: the impact of upstream and downstream integration. International Journal of Operations \& Production Management, v. 26, n. 7, p. 795-821, 2006.

VAN DER ZEE, D. J.; VAN DER VORST, J. G. A. J. A modeling framework for supply chain simulation: opportunities for improved decision making. Decision Sciences, v. 36, n. 1, p. 65-95, 2005.

VOORDIJK, H.; MEIJBOOM, B.; HAAN, J. Modularity in supply chains: a multiple case study in the construction industry. International Journal of Operations \& Production Management, v. 26, n. 6, p. 600-618, 2006.

WANG, H.; GUO, M.; EFSTATHIOU, J. A game-theoretical cooperative mechanism design for a two-echelon decentralized supply chain. European Journal of Operational Research, v. 157, p. 372-388, 2004.

WANG, S.; SARKER, B. R. Optimal models for a multi-stage supply chain system controlled by kanban under justin-time philosophy. European Journal of Operational Research, v. 172, p. 179-200, 2006.

WARBURTON, R. D. H. An analytical investigation of the bullwhip effect. Production and Operations Management, V. 13 , n. 2, p. 150-160, 2004

WASTI, S. N.; KOZAN, M. K.; KUMAN, A. Buyer-supplier relationship in the Turkish automotive industry. International Journal of Operations \& Production Management, v. 26, n. 9, p. 947-970, 2006.

WATSON, N.; ZHENG, Y. S. Decentralized serial supply chains subject to order delays and information distortion: exploiting real-time sales data. Manufacturing \& Service Operations Management, v. 7, n. 2, p. 152-168, 2005. 
WU, Z.; CHOI, T. Y. Supplier-supplier relationships in the buyer-supplier triad: building theories from eight case studies. Journal of Operations Management, v. 24, p. 27-52, 2005.

XIAO, T.; YU, G. Supply chain disruption management and evolutionarily stable strategies of retailers in the quantitysetting duopoly situation with homogeneous goods. European Journal of Operational Research, v. 173, p. 648-668, 2006.

YAO, M.; CHIOU, C. On a replenishment coordination model in an integrated supply chain with one vendor and multiple buyers. European Journal of Operational Research, v. 159, p. 406-419, 2004.

YUE, J. et al. Coordination of cooperative advertising in a two-level supply chain when manufacturer offers discounts. European Journal of Operational Research, v. 168, p. 65-85, 2006.

YUSUF, Y. Y. et al. Agile supply chain capabilities: determinants of competitive objectives. European Journal of Operational Research, v. 159, p. 379-392, 2004.

ZHOU, H.; BENTON Jr., W. C. Supply Chain Practice and Information Sharing. Journal of Operations Management, v. 27, p. 1-41, 2007.

ZHU, Q.; SARKIS, J.; GENG, Y. Green supply chain management in China: pressures, practices and performance. International Journal of Operations \& Production Management, v. 25, n. 5, p. 449-468, 2005. 


\section{Anexo II - Artigos Nacionais Analisados}

ALVES FILHO, A. G. et al. Pressupostos da gestão da cadeia de suprimentos: evidências de estudos sobre a indústria automobilística. Gestão \& Produção, v. 11, n. 3, p. 275-288, 2004.

ARAGÃO, A. B.; SCAVARDA, L. F.; HAMACHER, S. Modelo de análise de cadeias de suprimentos: fundamentos e aplicação às cadeias de cilindros de GNV. Gestão \& Produção, v. 11, n. 3, p. 299-311, 2004.

CABRAL, S. Analisando a reconfiguração da cadeia de produção de pneus no Brasil pela economia dos custos de transação. Gestão \& Produção, v. 11, n. 3, p. 373-384, 2004.

CONCEIÇÃO, S. V.; QUINTÃO, R. T. Avaliação do desempenho logistic da cadeia brasileira de suprimentos de refrigerantes. Gestão \& Produção, v. 11, n. 3, p. 441-453, 2004.

FURTADO, P. G.; CARVALHO, M. F. H. Compartilhamento da informação como element de coordenação da produção em cadeia de suprimento. Gestão \& Produção, v. 12, n. 1, p. 29-53, 2005.

GARCIA, R.; MOTTA, F. G.; AMATO NETO, J. Uma análise das características da estrutura de governança em sistemas locais de produção e suas relações com a cadeia global. Gestão \& Produção, v. 11, n. 3, p. 343-354, 2004.

LOURENZANI, A. E. B. S; SILVA, A. L. Um estudo da competitividade dos diferentes canais de distribuição de hortaliças. Gestão \& Produção, v. 11, n. 3, p. 385-398, 2004.

MAIA, J. L.; CERRA, A. L.; ALVES FILHO, A. G. Inter-relações entre a Estratégia de Operações e Gestão da Cadeia de Suprimentos: estudos de caso no segment de motores para automóveis. Gestão \& Produção, v. 12, n. 3, p. 377-391, 2005.

MARQUES, E. F.; ALCÂNTARA, R. L. C. O uso da ferramenta de gerenciamento por categoria na gestão da cadeia de suprimentos: um estudo multicaso. Gestão \& Produção, v. 11, n. 2, p. 153-164, 2004.

MARTINS, R. S. et al. Desenvolvimento de uma ferramenta para gestão logística da captação de leite de cooperativa agropecuária. Gestão \& Produção, v. 11, n. 3, p. 429-440, 2004.

MAZZALI, L.; MILAN, M. A integração empresa cliente - operador logistic: uma análise na cadeia automotiva. Gestão \& Produção, v. 13, n. 2, p. 353-366, 2006.

MORINI, C.; PIRES, S. R. I. Um modelo de decisão sobre consignação de material estrangeiro em cadeias de suprimento. Gestão \& Produção, v. 12, n. 1, p. 67-80, 2004.

OLIVEIRA, C. M.; CARVALHO, M. F. H. Análise das políticas de gestão em cadeias de suprimentos por modelos de simulação. Gestão \& Produção, v. 11, n. 3, p. 313-329, 2004.

ROLDAN, F.; MIYAKE, D. I. Mudanças no forecast na indústria automobilística: iniciativas para a estruturação dos processos de tomada de decisão e processamento da informação. Gestão \& Produção, v. 11, n. 3, p. 413-427, 2004.

SCRAMIM, F. C. L.; BATALHA, M. O. Método para análise de benefícios em cadeias de suprimento: um estudo de caso. Gestão \& Produção, v. 11, n. 3, p. 331-342, 2004.

TALAMINI, E.; PEDROZO, E. A.; SILVA, A. L. Gestão da cadeia de suprimentos e a segurança do alimento: uma pesquisa exploratória na cadeia exportadora de carne suína. Gestão \& Produção, v. 12, n. 1, p. 107-120, 2004.

TEIXEIRA, L.; NEVES, M. F.; SCARE, R. F. Auditoria e sistema de informação em canais de insumos do agronegócio: proposta de uma ferramenta de pesquisa. Gestão \& Produção, v. 11, n. 3, p. 399-411, 2004.

TOLEDO, J. C. et al. Coordenação da qualidade em cadeias de produção: estrutura e método para cadeias agroalimentares. Gestão \& Produção, v. 11, n. 3, p. 355-372, 2004.

VASCONCELOS, M. C. R. L; MILAGRES, R.; NASCIMENTO, E. Estratégia de relacionamento entre os membros da cadeia produtiva no Brasil: reflexões sobre o tema. Gestão \& Produção, v. 12, n. 3, p. 393-404, 2005.

WANKE, P. Impactos da sofisticação logística de empresas industriais nas motivações para terceirização. Gestão \& Produção, v. 11, n. 3, p. 455-467, 2004.

WANKE, P.; SALIBY, E. Proposta para a gestão de estoques de novos produtos: solução do modelo (Q,r) para distribuição uniforme da demanda e do lead-time de suprimento. Gestão \& Produção, v. 12, n. 1, p. 1-9, 2005.

WANKE, P. et al. Um estudo sobre os impactos no varejo das principais decisões estratégicas de produção e distribuição da indústria. Gestão \& Produção, v. 13, n. 1, p. 1-13, 2006.

ZHIXIANG, C. Investigation of supplier/buyer coordination performance in Chinese companies. Gestão \& Produção, v. 11, n. 3, p. 289-298, 2004. 